AnD: A Many-Objective Evolutionary Algorithm with Angle-based Selection and Shift-based Density Estimation

Zhi-Zhong Liu, Yong Wang, Pei-Qiu Huang

PII: S0020-0255(18)30512-7

DOI: 10.1016/j.ins.2018.06.063

Reference: INS 13756

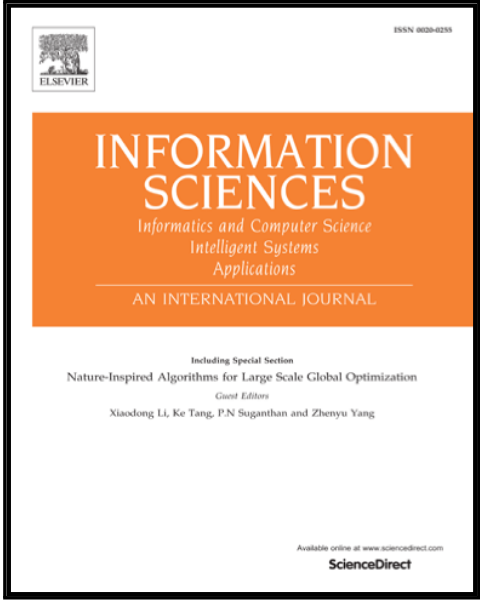

To appear in:

Information Sciences

Received date:

17 April 2018

Revised date:

21 June 2018

Accepted date:

29 June 2018

Please cite this article as: Zhi-Zhong Liu, Yong Wang, Pei-Qiu Huang, AnD: A Many-Objective Evolutionary Algorithm with Angle-based Selection and Shift-based Density Estimation, Information Sciences (2018), doi: 10.1016/j.ins.2018.06.063

This is a PDF file of an unedited manuscript that has been accepted for publication. As a service to our customers we are providing this early version of the manuscript. The manuscript will undergo copyediting, typesetting, and review of the resulting proof before it is published in its final form. Please note that during the production process errors may be discovered which could affect the content, and all legal disclaimers that apply to the journal pertain. 


\section{Highlights}

- We design AnD as an alternative MaOEA, which has a simple structure, few parameters, and no complicated operators. More importantly, AnD is different from existing methodsit does not use dominance rules, weight vectors/reference points, and indicators.

- To the best of our knowledge, it is the first attempt to effectively combine vector angle with shift-based density estimation for solving MaOPs, by making use of their complementary properties.

- We compared AnD with other seven state-of-the-art MaOEAs on a variety of benchmark test problems with up to 15 objectives. The results provide evidence that $\mathrm{AnD}$ can achieve highly competitive performance.

- AnD has been further extended to solve constrained MaOPs with promising performance 


\title{
AnD: A Many-Objective Evolutionary Algorithm with Angle-based Selection and Shift-based Density Estimation
}

\author{
Zhi-Zhong Liu ${ }^{\mathrm{a}}$, Yong Wanga,b,*, Pei-Qiu Huang ${ }^{\mathrm{a}}$ \\ ${ }^{a}$ School of Information Science and Engineering, Central South University, Changsha 410083, \\ China. \\ ${ }^{b}$ School of Computer Science and Electronic Engineering, University of Essex, Colchester CO4
} $3 S Q, U K$.

\begin{abstract}
Evolutionary many-objective optimization has been gaining increasing attention from the evolutionary computation research community. Much effort has been devoted to addressing this issue by improving the scalability of multiobjective evolutionary algorithms, such as Pareto-based, decomposition-based, and indicatorbased approaches. Different from current work, we propose an alternative algorithm in this paper called $\mathrm{AnD}$, which consists of an angle-based selection strategy and a shift-based $d$ ensity estimation strategy. These two strategies are employed in the environmental selection to delete poor individuals one by one. Specifically, the former is devised to find a pair of individuals with the minimum vector angle, which means that these two individuals have the most similar search directions. The latter, which takes both diversity and convergence into account, is adopted to compare these two individuals and to delete the worse one. AnD has a simple structure, few parameters, and no complicated operators. The performance of AnD is compared with that of seven state-of-the-art many-objective evolutionary algorithms on a variety of benchmark test problems with up to 15 objectives. The results/suggest that $\mathrm{AnD}$ can achieve highly competitive performance. In addition, we also verify that $\mathrm{AnD}$ can be readily extended to solve constrained many-objective optimization problems.
\end{abstract}

Keywords: Evolutionary algorithms, many-objective optimization, angle-based

${ }^{*}$ Corresponding author.

Email address: ywang @esu . edu. cn (Yong Wang) 
selection, shift-based density estimation

\section{Introduction}

Multiobjective optimization problems (MOPs) refer to optimization problems with more than one conflicting objective. Usually, a MOP can be expressed as:

$$
\begin{aligned}
& \text { minimize } \mathbf{F}(\mathbf{x})=\left(f_{1}(\mathbf{x}), f_{2}(\mathbf{x}), \ldots, f_{m}(\mathbf{x})\right) \\
& \text { subject to } \mathbf{x} \in \Omega
\end{aligned}
$$

where $\mathbf{x}=\left(x_{1}, x_{2}, \ldots, x_{n}\right)$ is the decision vector, $n$ is the number of decision variables, $\mathbf{F}(\mathbf{x})$ is the objective vector, $m$ is the number of objectives, and $\Omega$ is the decision space. The ultimate goal of multiobjective optimization is to obtain a set of well-distributed and well-converged nondominated solutions to approximate the Pareto front (PF). To achieve this goal, numerous multiobjective evolutionary algorithms (MOEAs) have been proposed over the last few decades. According to their selection mechanisms, MOEAs can be roughly classified into three categories: Pareto-based methods, decomposition-based methods, and indicatorbased methods [44]. MOEAs have shown great potential to solve MOPs with two or three objectives. However, for MOPs with more than three objectives, often known as many-objective optimization problems (MaOPs), they encounter substantial difficulties [19].

For Pareto-based methods, such as NSGA-II [9] and SPEA2 [46], the selection criteria (i.e., the Pareto-based selection and the diversity-based selection) may lose their effectiveness to push the population toward the PF. This is because with an increase in the number of objectives, the proportion of nondominated solutions increases drastically. As a result, the Pareto-based (primary) selection fails to distinguish the individuals in the population. Under this condition, the diversity-based (secondary) selection will play a major role in the selection process. The secondary selection may distribute the population well over the objective space; however, the population tends to be far away from the desired PF due to the neglect of convergence performance. Decomposition-based [42] and indicator-based [2] methods do not suffer from selection pressure issues since they do not rely on Pareto dominance to evolve the population. However, they face their own challenges. Regarding decomposition-based methods, it is not a trivial task to assign the weight vectors or reference points in the high-dimensional objective space [22]. In addition, indicator-based methods always result in high computational time complexity [33]. 
To enhance the scalability of MOEAs for MaOPs, a considerable number of attempts have been made to improve the performance of Pareto-based, decompositionbased, and indicator-based methods. These are briefly introduced next.

- Pareto-based Methods: Recognizing the drawback of the Pareto-dominance relation for MaOPs, this kind of method intends to modify/relax the definition of Pareto dominance. Along this line, several rules have been proposed such as $\epsilon$-dominance [23], L-dominance [48], and fuzzy dominance [34]. Additionally, another avenue is to develop customized diversity mechanisms, with the purpose of alleviating the loss of selection pressure. In [1], a diversity management mechanism is introduced, which can determine whether or not to activate diversity promotion based on the distribution of population. In [27], a shift-based density estimation strategy is proposed, which shifts the poorly converged individuals into crowded regions and assigns them high density values. As a result, these individuals are very likely to be removed from the population. Inspired by the idea that the knee points are naturally most preferred among nondominated solutions, a knee pointdriven EA is proposed in [43], in which diversity is embedded in the knee point identification process.

- Decomposition-based Methods: This kind of method contains two different types. The first type decomposes a MaOP into a series of single-objective optimization problems. MOEA/D [42] is the most famous one. In MOEA/D, a set of weight vectors are predefined to specify multiple search directions toward the PF. Since the search directions spread out widely, it is expected that the obtained solutions cover the PF well. MOEA/D was originally designed for solving MOPs. Recent advances have successfully adapted MOEA/D to solve MaOPs. Examples include adaptively allocating search effort in MOEA/D-AM2M [28], exploiting the perpendicular distance from the solution to the weight vector in MOEA/D-DU [41], and using Pareto adaptive scalarizing methods in MOEA/D-PaS [38]. The second type divides a MaOP into a group of sub-MaOPs. One representative is NSGAIII [8], which makes use of a set of predefined well-distributed reference points to manage nondominated solutions. That is, the nondominated solutions close to the reference points are prioritized. For these two types, to achieve good performance, a crucial issue is how to assign the appropriate weight vectors or reference points. To this end, an automatic weight vector generation system is devised in [18], and a two-layered generation strategy for reference points is proposed in [8]. 
- Indicator-based Methods: In this kind of method, the indicator values are used to guide the search process. Among all the indicators, the hypervolume indicator [47] is the most commonly used, which is originally a quality indicator to compare different MOEAs. The hypervolume indicator has an attractive property, that is, it is strictly monotonic with regard to Pareto dominance [2]. Note, however, that the burden for calculating hypervolume is very high, and increases exponentially as the number of objectives increases. To overcome this shortcoming, the Monte Carlo simulation is employed in [2] to approximate the exact hypervolume values, with the aim of striking a tradeoff between accuracy and computational time. Additionally, there are some cheap indicators, such as the $I_{(\epsilon)}+$ indicator in IBEA [45] and the $R 2$ indicator in R2-EMOA [32]. The collaboration of different cheap indicators seems to be a promising direction for solving MaOPs [25].

Apart from these three categories, several preference-based many-objective EAs (MaOEAs) have been proposed recently $[37,13]$ which focus on a subset of the $\mathrm{PF}$ based on the user's preference. There are also some dimensionality reduction approaches [30, 29, 14], aiming to deal with MaOPs with redundant objectives. Additionally, researchers have tried to take advantage of the merits offered by different categories. Two representatives are MOEA/DD and Two_Arch2. MOEA/DD [26] is based on Pareto dominance and decomposition, and Two_Arch2 [35] is based on Pareto dominance and an indicator. For more information about MaOEAs, interested readers are referred to a survey paper [24].

Unlike recent work, we propose an alternative MaOEA, called AnD. In evolutionary many-objective optimization, the task of environmental selection is to choose some promising individuals from the union population, which is composed of the parent and offspring populations, for the next generation. AnD tackles this task by two strategies: angle-based selection and shift-based density estimation. First, angle-based selection finds a pair of individuals with the minimum vector angle. Intuitively, it is necessary to delete one of these two individuals since they search in the most similar directions and it will waste significant computational resources if they coexist. In order to make the deletion wiser, we need to take both convergence and diversity into account since achieving balance between convergence and diversity is the most important concern in many-objective optimization. Fortunately, shift-based density estimation has the capability to cover both the distribution and convergence information of individuals [27]. Therefore, it is utilized to compare these two individuals and to delete the worse one. By repeating this process, AnD provides a quite natural way for solving MaOPs - the individuals 
with poor diversity and convergence are eliminated from the union population one by one.

The main contributions of this paper are summarized as follows:

- We design AnD as an alternative MaOEA that has a simple structure, few parameters, and no complicated operators. More importantly, AnD is different from existing methods because it does not use dominance rules, weight vectors/reference points, and indicators. As a consequence, it has the following advantages for solving MaOPs: no disadvantages incurred from insufficient selection pressure as in Pareto-based methods, no need to assign weight vectors/reference points as in decomposition-based methods, and no need to consume a high computational cost as in indicator-based methods.

- The vector angle $[4,40]$ and shift-based density estimation $[27,36]$ have been extensively investigated in the design of MaOEAs, respectively. However, to the best of our knowledge, ours is the first attempt to effectively combine them together for solving MaOPs, by making use of their complementary properties. Moreover, AnD provides a straightforward way to achieve both diversity and convergence - by identifying the two individuals with the minimum vector angle via angle-based selection and removing the one with worse diversity and convergence via shift-based density estimation in an iterative way.

- Systematic experiments haye been conducted on both the DTLZ and WFG test suites to demonstrate the effectiveness of AnD. The performance of AnD is compared with that of seven state-of-the-art MaOEAs. The experimental results suggest that, overall, $\mathrm{AnD}$ can achieve better performance in terms of two widely used performance metrics: IGD [5] and HV [47].

- AnD has been further extended to solve constrained MaOPs with promising performance.

The rest of this paper is organized as follows. Section 2 introduces preliminary knowledge. The details of AnD are presented in Section 3. Subsequently, the experimental setup is described in Section 4. The empirical results on both unconstrained and constrained MaOPs are given in Section 5. Finally, Section 6 concludes this paper. 


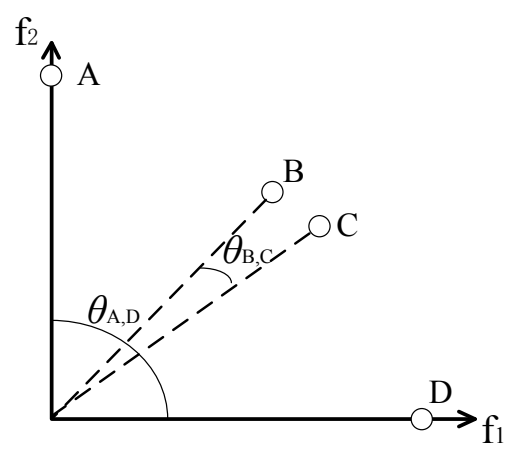

Figure 1: Illustration of the vector angle in a bi-objective minimization scenario

\section{Preliminary Knowledge}

\subsection{Vector Angle}

In this paper, the vector angle denotes the included angle between two individuals in the normalized objective space. The normalized objective vector of an individual is computed as follows. First, we find the ideal point $Z^{\text {min }}=$ $\left(z_{1}^{\min }, z_{2}^{\min }, \ldots, z_{m}^{\min }\right)$ and estimate the nadir point as $Z^{\max }=\left(z_{1}^{\max }, z_{2}^{\max }, \ldots, z_{m}^{\max }\right)$, where $z_{i}^{\min }$ and $z_{i}^{\max }$ are the minimum and maximum values of the $i$ th objective of all individuals, respectively. Afterward, for the $j$ th individual $\mathbf{x}_{j}$, its objective vector $\mathbf{F}\left(\mathbf{x}_{j}\right)$ is normalized as $\mathbf{F}^{\prime}\left(\mathbf{x}_{j}\right)=\left(f_{1}^{\prime}\left(\mathbf{x}_{j}\right), f_{2}^{\prime}\left(\mathbf{x}_{j}\right), \ldots, f_{m}^{\prime}\left(\mathbf{x}_{j}\right)\right)$ according to

$$
f_{i}^{\prime}\left(\mathbf{x}_{j}\right)=\frac{f_{i}\left(\mathbf{x}_{j}\right)-z_{i}^{\min }}{z_{i}^{\text {max }}-z_{i}^{\min }}, i=1,2, \ldots, m
$$

After the normalization, the vector angle between two individuals $\mathbf{x}_{j}$ and $\mathbf{x}_{l}$, referred to as $\theta_{\mathbf{x}_{j}, \mathbf{x}_{l}}$, is computed as

$$
\theta_{\mathbf{x}_{j}, \mathbf{x}_{l}}=\arccos \left|\frac{\mathbf{F}^{\prime}\left(\mathbf{x}_{j}\right) \bullet \mathbf{F}^{\prime}\left(\mathbf{x}_{l}\right)}{\left\|\mathbf{F}^{\prime}\left(\mathbf{x}_{j}\right)\right\| \times\left\|\mathbf{F}^{\prime}\left(\mathbf{x}_{l}\right)\right\|}\right| .
$$

where $\mathbf{F}^{\prime}\left(\mathbf{x}_{j}\right) \bullet \mathbf{F}^{\prime}\left(\mathbf{x}_{l}\right)$ denotes the inner product of $\mathbf{F}^{\prime}\left(\mathbf{x}_{j}\right)$ and $\mathbf{F}^{\prime}\left(\mathbf{x}_{l}\right)$, and $\|\cdot\|$ calculates the norm of a vector. It is clear that $\theta_{\mathbf{x}_{j}, \mathbf{x}_{l}} \in\left[0, \frac{\pi}{2}\right]$.

In principle, the vector angle reflects the similarity of search directions between two individuals. To be specific, if two individuals search in quite different directions, the vector angle between them is large; otherwise, the vector angle is 
small. Fig. 1 gives an example. From Fig. 1, we can observe that: 1) individuals $\mathbf{A}$ and $\mathbf{D}$ search in quite different directions, and $\theta_{\mathbf{A , D}, \mathbf{D}}$ is relatively larger; and 2) individuals $\mathbf{B}$ and $\mathbf{C}$ share similar search directions, and $\theta_{\mathbf{B}, \mathbf{C}}$ is relatively smaller.

During the past two years, the vector angle has attracted a high level of interest for evolutionary many-objective optimization. For instance, it has been incorporated into decomposition-based approaches. In [4], a reference-vector-guided EA (RVEA) for many-objective optimization is proposed. In RVEA, the anglepenalized distance is used to balance the convergence and diversity of individuals in the high-dimensional objective space. In [39], a novel decomposition-based MaOEA called MOEA/D-LWS is proposed. In MOEA/D-LWS, for each search direction, the optimal solution is selected only among its neighboring solutions. Note that the neighborhood is defined by a hypercone, whose apex angle is determined automatically a priori. Very recently, a new variant of MOEA/D with sorting-and-selection (MOEA/D-SAS) has been presented [3]. In MOEA/D-SAS, the balance between convergence and diversity is achieved by two distinctive components: decomposition-based sorting and angle-based selection. In the latter, the angle information between two individuals in the objective space is used to maintain diversity. In addition, the vector angle also has the potential to improve the performance of Pareto-based approaches. In [40], a vector-angle-based EA (VaEA) for unconstrained many-objective optimization is developed. VaEA implements the nondominated sorting procedure to obtain different layers, and deals with the last layer through the vector angle.

Other kinds of attempts have also been made to solve MaOPs with the use of the vector angle. For example, He and Yen [15] suggested a MaOEA based on a coordinate selection strategy (MaOEA-CSS), in which a new diversity measure based on the vector angle is designed in the mating and environmental selection.

\subsection{Shift-based Density Estimation}

Shift-based density estimation is an advanced density estimation strategy proposed by Li et al. [27]. Compared with traditional density estimation, it shifts the positions of other individuals when estimating the density of an individual (e.g., $\mathbf{x}_{j}$ ) in the population $\mathcal{P}$. This shift process is simple and is based on the convergence comparison between other individuals and $\mathbf{x}_{j}$ on each objective. To be specific, if $\mathbf{x}_{l}$ (suppose that $\mathbf{x}_{l}$ is another individual in $\mathcal{P}$ ) outperforms $\mathbf{x}_{j}$ on one objective, its objective value on this objective will be shifted to the same position of $\mathbf{x}_{j}$ on this objective; otherwise, its objective value remains unchanged. This 


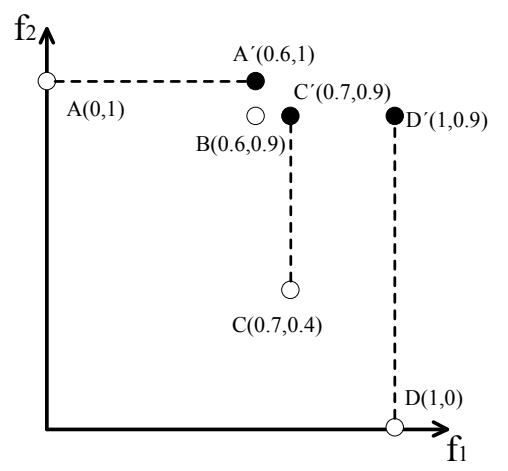

Figure 2: Illustration of shift-based density estimation in a two-dimensional normalized objective space. To estimate the density of individual $\mathbf{B}$, the other individuals $\mathbf{A}, \mathbf{C}$, and $\mathbf{D}$ are shifted to $\mathbf{A}^{\prime}$, $\mathbf{C}^{\prime}$, and $\mathbf{D}^{\prime}$, respectively.

shift process can be described as

$$
f_{i}^{s}\left(\mathbf{x}_{l}\right)=\left\{\begin{array}{l}
f_{i}^{\prime}\left(\mathbf{x}_{j}\right), \text { if } f_{i}^{\prime}\left(\mathbf{x}_{l}\right)<f_{i}^{\prime}\left(\mathbf{x}_{j}\right) \\
f_{i}^{\prime}\left(\mathbf{x}_{l}\right), \text { otherwise }
\end{array}\right.
$$

where $f_{i}^{s}\left(\mathbf{x}_{l}\right)$ is the shifted objective value of $f_{i}^{\prime}\left(\mathbf{x}_{l}\right)$, and $\mathbf{F}^{s}\left(\mathbf{x}_{l}\right)=\left(f_{1}^{s}\left(\mathbf{x}_{l}\right), f_{2}^{s}\left(\mathbf{x}_{l}\right)\right.$, $\left.\ldots, f_{m}^{s}\left(\mathbf{x}_{l}\right)\right)$ is the shifted objective vector of $\mathbf{F}^{\prime}\left(\mathbf{x}_{l}\right)$. Note that before shifting, the objective vector of each individual is normalized via Eq. (2).

To understand the shift process more clearly, we take shift-based density estimation of individual $\mathbf{B}(0.6,0.9)$ in Fig. 2 as an example. First, individuals $\mathbf{A}(0,1), \mathbf{C}(0.7,0.4)$, and $\mathbf{D}(1,0)$ in Fig. 2 are shifted to individuals $\mathbf{A}^{\prime}(0.6,1)$, $\mathbf{C}^{\prime}(0.7,0.9)$, and $\mathbf{D}^{\prime}(1,0.9)$, respectively, due to the fact that $\mathbf{A}_{1}=0<\mathbf{B}_{1}=0.6$, $\mathbf{C}_{2}=0.4<\mathbf{B}_{2}=0.9$, and $\mathbf{D}_{2}=0<\mathbf{B}_{2}=0.9$. Subsequently, it can be observed that the poorly converged individual $\mathbf{B}$ is located in a crowded region. Thus, $\mathbf{B}$ will be assigned a high density value and is very likely to be removed from the population. It is noteworthy that in order to obtain the density of an individual, the shift process should be combined with a density estimator, such as the crowding distance in NSGA-II [9], the $k$ th nearest neighbor in SPEA2 [46], or the grid crowding degree in PESA-II [6]. Actually, as pointed out in [27], only the individual with both good diversity and good convergence will have a low density value, which means that both diversity and convergence are elaborately considered in the shift-based density estimation strategy. 
In this paper, we integrate shift-based density estimation with the $k$ th nearest neighbor to estimate the density of individual $\mathbf{x}_{j}$ in $\mathcal{P}$, denoted as $S D\left(\mathbf{x}_{j}\right)$. The implementation is the following:

1. Shift the normalized objective vectors of the other individuals in $\mathcal{P}$ via Eq. (4);

2. Calculate the Euclidian distances between the other shifted normalized objective vectors and $\mathbf{F}^{\prime}\left(\mathbf{x}_{j}\right)$ according to:

$$
d\left(\mathbf{x}_{j}, \mathbf{x}_{l}\right)=\left\|\mathbf{F}^{s}\left(\mathbf{x}_{l}\right)-\mathbf{F}^{\prime}\left(\mathbf{x}_{j}\right)\right\|, \mathbf{x}_{l} \in \mathcal{P} \cap \mathbf{x}_{l} \neq \mathbf{x}_{j}
$$

3. Find the $k$ th minimum value $\ell\left(\mathbf{x}_{k}\right)$ in the set of $\left\{d\left(\mathbf{x}_{j}, \mathbf{x}_{l}\right), \mathbf{x}_{l} \in \mathcal{P} \cap \mathbf{x}_{l} \neq \mathbf{x}_{j}\right\}$, where $k$ is set to $\sqrt{N}$ and $N$ is the size of $\mathcal{P}$;

4. Compute $S D\left(\mathbf{x}_{j}\right)$ according to Eq. (6):

$$
S D\left(\mathbf{x}_{j}\right)=\frac{1}{\ell\left(\mathbf{x}_{k}\right)+2} .
$$

Note that the higher the density value, the worse the performance of an individual.

The shift-based density estimation strategy has become an important technique in evolutionary many-objective optimization. From [27], it can significantly enhance the scalability of NSGA-II [9], SPEA2 [46], and PESA2 [6] for solving MaOPs. Moreover, SPEA2 achieves better performance than NSGA-II and PESA2 after these three algorithms are integrated with shift-based density estimation. Recently, Wang et al. [36] presented a cooperative differential evolution with multiple populations (CMODE) for multi- and many-objective optimization. From the results, the combination of CMODE and shift-based density estimation reaches outstanding performance when solving MaOPs. Very recently, Li et al. [25] presented a stochastic ranking-based multi-indicator algorithm (SRA). SRA adopts the stochastic ranking technique to balance the search biases of different indicators. Among these indicators, one is designed based on shift-based density estimation.

\section{Proposed Approach}

\section{1. $A n D$}

The framework of AnD is given in Algorithm 1. First, a population $\mathcal{P}_{0}$ with $N$ individuals is randomly initialized in the decision space $\Omega$. During the evolution, an offspring population $\mathcal{Q}_{t}$ is generated from $\mathcal{P}_{t}$ through mating. Afterward, 


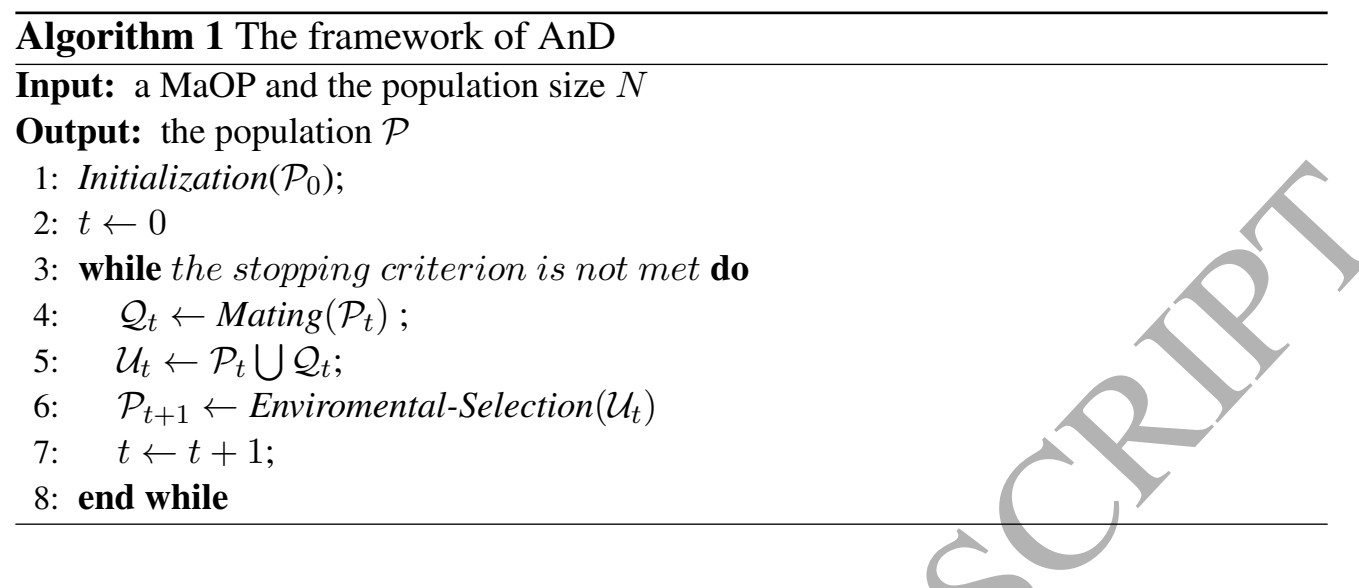

a union population $\mathcal{U}_{t}$ is obtained by combining $\mathcal{Q}_{t}$ with $\mathcal{P}_{t}$. Finally, the environmental selection is performed on $\mathcal{U}_{t}$ to produce the next population $\mathcal{P}_{t+1}$. The above procedure repeats until the stopping criterion is met.

It can be seen that similar to most MaOEAs, AnD involves two main components: mating and environmental selection. The aim of mating is to generate a number of offspring (i.e., $\mathcal{Q}_{t}$ ) from the parents (i.e., $\mathcal{P}_{t}$ ) by making use of evolutionary operators, such as selection, crossover, and mutation. AnD does not apply any explicit selection to choose parents from $\mathcal{P}_{t}$ or employ any special crossover and mutation to generate offspring. Instead, the parents are randomly chosen from $\mathcal{P}_{t}$, and the simulated binary crossover (SBX) and the polynomial mutation are utilized to generate $\mathcal{Q}_{t}$. The reasons are twofold: 1) the random selection, SBX, and the polynomial mutation have been widely used in the community of evolutionary many-objective optimization, and 2) we would like to ensure a fair comparison with other algorithms. The unique characteristic of AnD lies in its environmental selection, which is described in the sequel.

\subsection{Environmental Selection}

The environmental selection aims at choosing $N$ individuals with the most potential from the union population $\mathcal{U}_{t}$ for the next generation. AnD accomplishes this by two strategies: angle-based selection and shift-based density estimation. Algorithm 2 describes the environmental selection of AnD. Firstly, the vector angles between any two individuals in $\mathcal{U}_{t}$ are calculated. Thereafter, angle-based selection is conducted to identify two individuals (denoted as $\mathbf{u}_{j}$ and $\mathbf{u}_{l}$ ) with the minimum vector angle in $\mathcal{U}_{t}$. Subsequently, shift-based density estimation is employed to compare $\mathbf{u}_{j}$ and $\mathbf{u}_{l}$, and the one with a higher density value is 


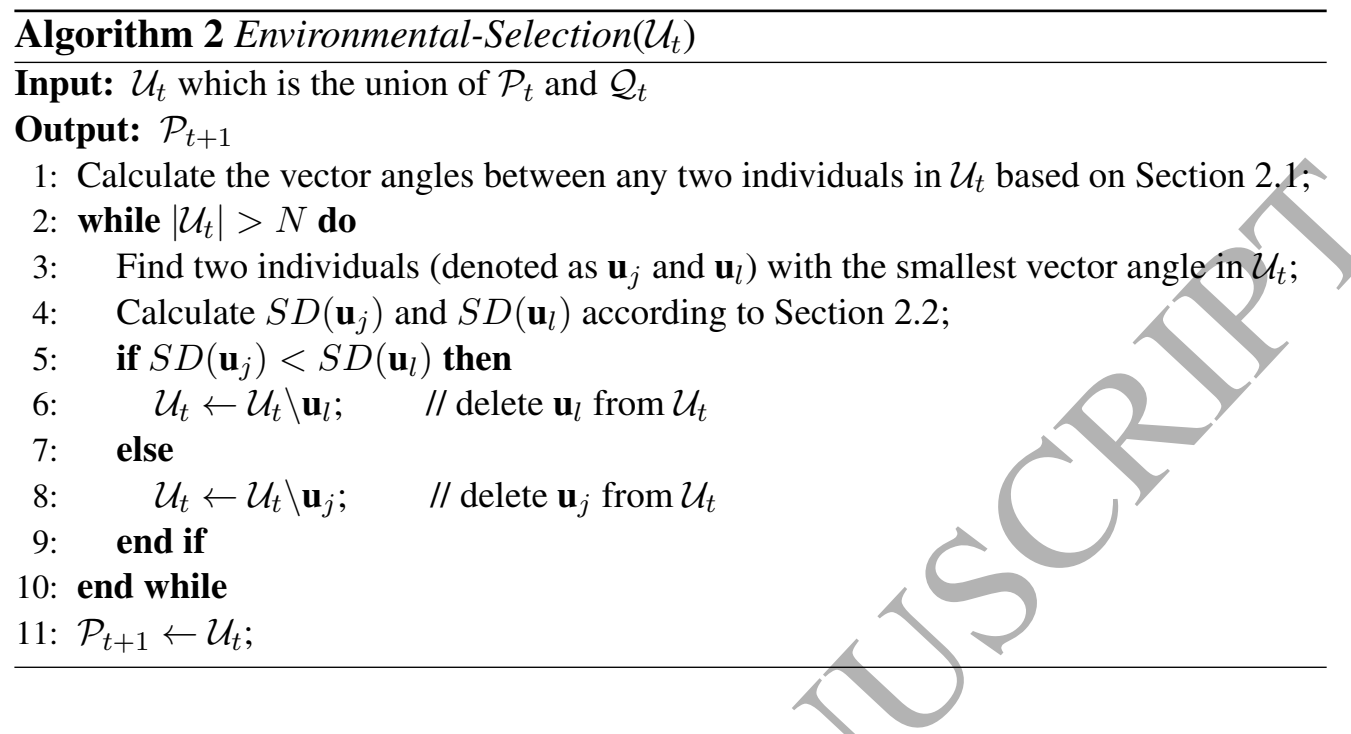

removed from $\mathcal{U}_{t}$. This process proceeds until the size of $\mathcal{U}_{t}$ is equal to $N$. Next, we explain the importance of the combination of these two strategies in AnD.

As mentioned in Section 2.1, the yector angle reflects the similarity between two individuals in their search directions. If two individuals share the minimum vector angle, they absolutely have the most similar search directions. To improve the diversity of search directions, one of them should be discarded. If we repeatedly delete one of two individuals with the most similar search directions in the remaining population, the diyersity of search directions will be well maintained in the final population. Indeed, the main idea behind angle-based selection is to approximate the PF from diverse search directions. Decomposition-based approaches, which have shown great success in solving MaOPs, also use a similar idea. However, compared with decomposition-based approaches, angle-based selection only exploits the information provided by the vector angle.

Although angle-based selection is able to find a pair of individuals with the most similar search directions, it cannot distinguish them. In many-objective optimization, when comparing two individuals, it has been widely accepted that both diversity and convergence should be considered. Fortunately, shift-based density estimation provides an effective way to measure the quality of two individuals because it focuses on both the diversity and convergence of individuals as introduced in Section 2.2. As a result, shift-based density estimation has the capability to judge which individual is worse and should be deleted under this condition.

Overall, the environmental selection of AnD combines angle-based selection and shift-based density estimation in a quite natural way: the former identifies the 


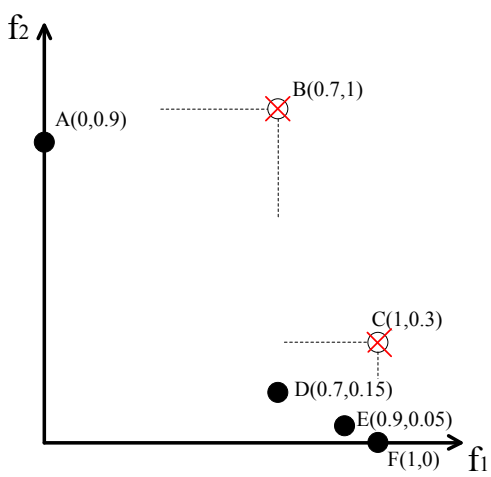

(a) NSGA-III and SPEA2+SDE

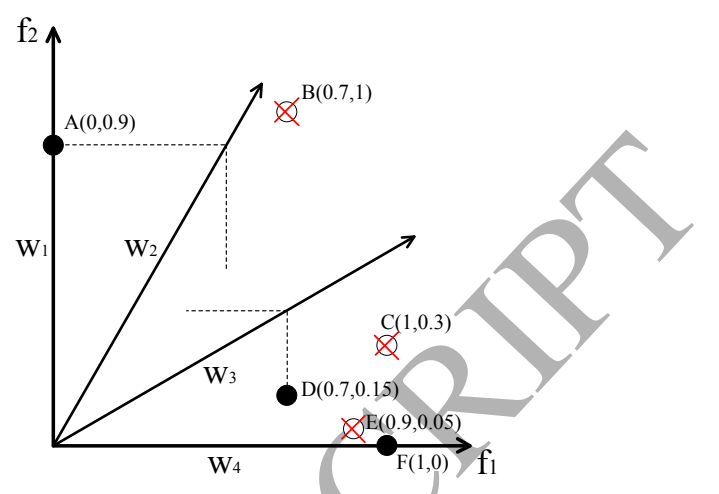

(b) MOEA/D

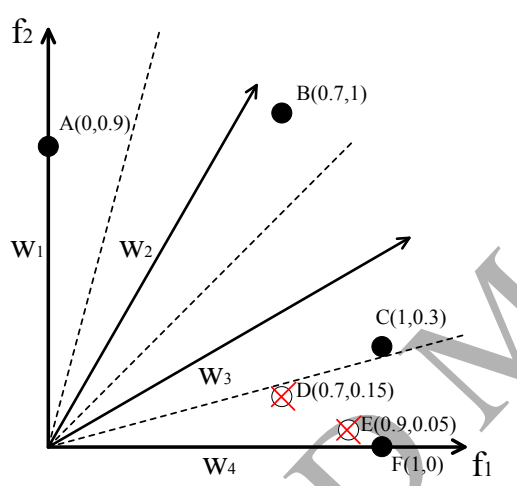

(c) MOEA/DD

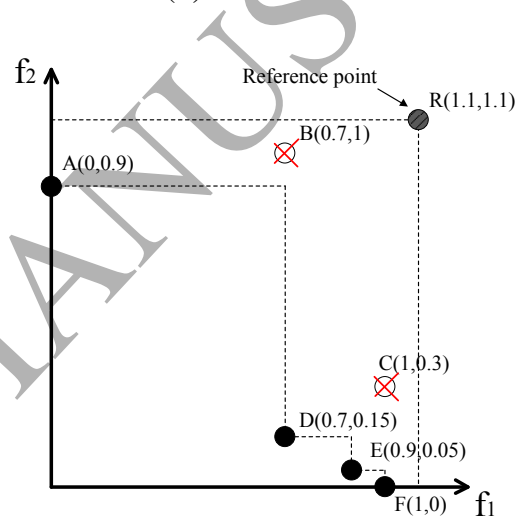

(d) HypE
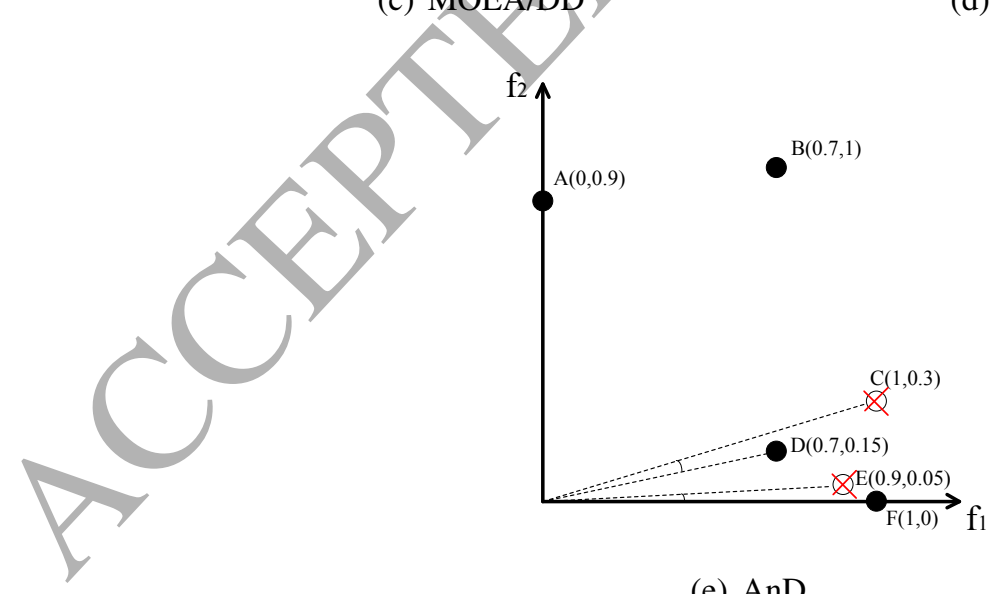

(e) AnD

Figure 3: Illustration of the working principles of six MaOEAs. There are six individuals in the population, i.e., $\mathbf{A}, \mathbf{B}, \mathbf{C}, \mathbf{D}, \mathbf{E}$, and $\mathbf{F}$ and the task is to select four promising individuals for the next generation. 
two most similar individuals in terms of search direction, and the latter eliminates the worse one in terms of both diversity and convergence. Therefore, by iteratively implementing both of them, the individuals with poor diversity and convergence are deleted one by one from $\mathcal{U}_{t}$; thus, the population continuously approaches the PF with good diversity.

\subsection{Analysis of the Principle}

An example in a two-dimensional objective space is used to illustrate the working principles of six MaOEAs: NSGA-III [8], SPEA2+SDE (a combination of SPEA2 and shift-based density estimation) [27], MOEA/D [42], MOEA/DD [26], HypE [2], and AnD. Suppose that there are six individuals (i.e., $\mathbf{A}(0,0.9), \mathbf{B}(0.7,1)$, $\mathbf{C}(1,0.3), \mathbf{D}(0.7,0.15), \mathbf{E}(0.9,0.05)$, and $\mathbf{F}(0,1))$ in the population, and our task is to select four promising individuals for the next generation. Fig. 3 depicts what happens to the six compared methods.

From Fig. 3, we can give the following comments:

- In both NSGA-III and SPEA2+SDE, A, D, E, and F are selected for the next generation. The reason is that these two algorithms prefer nondominated individuals. From Fig. 3(a), it can be observed that B is Pareto dominated by $\mathbf{A}$ and $\mathbf{D}$, and $\mathbf{C}$ is Pareto dominated by $\mathbf{D}, \mathbf{E}$, and $\mathbf{F}$. Thus, $\mathbf{A}, \mathbf{D}, \mathbf{E}$, and $\mathbf{F}$ are the nondominated individuals, while $\mathbf{B}$ and $\mathbf{C}$ are the dominated individuals.

- With respect to MOEA/D, suppose that there are four weight vectors (i.e., $\mathbf{w}_{1}, \mathbf{w}_{2}, \mathbf{w}_{3}$, and $\mathbf{w}_{4}$ ) as shown in Fig. 3(b), and that the Tchebycheff approach is used. According to the principle of MOEA/D with the Tchebycheff approach, each weight vector is associated with an individual. From Fig. 3(b), it is clear that $\mathbf{w}_{1}$ and $\mathbf{w}_{2}$ are associated with $\mathbf{A} ; \mathbf{w}_{3}$ is associated with $\mathbf{D}$; and $\mathbf{w}_{4}$ is associated with $\mathbf{F}$. Therefore, $\mathbf{A}, \mathbf{D}$ and $\mathbf{F}$ will survive into the next generation. In particular, $\mathbf{A}$ will be duplicated. The other individuals (i.e., $\mathbf{B}, \mathbf{C}$, and $\mathbf{E}$ ) will be eliminated.

- For MOEA/DD, $\mathbf{A}, \mathbf{B}, \mathbf{C}$, and $\mathbf{F}$ will survive into the next generation. This is because the weight vectors in MOEA/DD are used to divide the objective space into a series of subregions. From Fig. 3(c), we can see that A, B, and $\mathbf{C}$ are in their own isolated subregions, and all of them will be selected into the next generation. D, E, and $\mathbf{F}$ are in the same subregion, and only the boundary individual $\mathbf{F}$ will be chosen for the next generation. 
- In terms of HypE, like NSGA-III and SPEA2+SDE, $\mathbf{A}, \mathbf{D}, \mathbf{E}$, and $\mathbf{F}$ remain, and the others are deleted. The reason is because $\mathbf{B}$ and $\mathbf{C}$ do not contribute to the whole population's hypervolume value, while the other individuals (i.e., $\mathbf{A}, \mathbf{D}, \mathbf{E}$, and $\mathbf{F}$ ) do contribute. As a result, $\mathbf{B}$ and $\mathbf{C}$ are eliminated from the population.

- To implement AnD, firstly, it is necessary to calculate the vector angles between any two individuals in the population. Subsequently, we need to identify two individuals with the minimum vector angle and employ the shiftbased density estimation strategy to differentiate them. It is easy to find that $\theta_{\mathbf{E}, \mathbf{F}}$ is the minimum vector angle in the population. Then, according to Section 2, we can obtain the density values of $\mathbf{E}$ and $\mathbf{F}$ as $S D(\mathbf{E}) \approx 0.4762$ and $S D(\mathbf{F}) \approx 0.4651$, respectively. Thus, $\mathbf{E}$ will be removed from the population since it has the higher density value. After $\mathbf{E}$ has been eliminated, $\theta_{\mathbf{C , D}}$ becomes the minimum vector angle and the density values of $\mathbf{C}$ and $\mathbf{D}$ are computed as $S D(\mathbf{C})=0.5$ and $S D(\mathbf{D}) \approx 0.4348$, respectively. Thereafter, $\mathbf{C}$ will be removed from the population due to its higher density value. In summary, $\mathbf{C}$ and $\mathbf{E}$ will be eliminated from the population, while $\mathbf{A}, \mathbf{B}, \mathbf{D}$, and $\mathbf{F}$ will be chosen for the next generation.

From the above discussions, we can observe that:

- The principle of AnD is different from that of the other five state-of-the-art MaOEAs. AnD employs two simple strategies, namely angle-based selection and shift-based density estimation, to delete the inferior individuals one by one from the population.

- AnD can obtain more suitable results compared with the five competitors. In NSGA-HI, SPEA2+SDE, and HypE, A, D, E, and $\mathbf{F}$ survive into the next generation. In terms of MOEA/D, $\mathbf{A}, \mathbf{D}$, and $\mathbf{F}$ remain. In particular, $\mathbf{A}$ is copied. With respect to MOEA/DD, A, B, C, and $\mathbf{F}$ are chosen for survival. However, only in AnD, $\mathbf{A}, \mathbf{B}, \mathbf{D}$, and $\mathbf{F}$ are selected for the next generation simultaneously. Note that $\mathbf{B}$ plays an important role in maintaining the diversity of search directions since it is in a sparse region. Nevertheless, only in AnD and MOEA/DD, it survives. In contrast to MOEA/DD, AnD keeps $\mathbf{D}$ and deletes $\mathbf{C}$. This seems more reasonable since $\mathbf{C}$ and $\mathbf{D}$ share similar search directions but $\mathbf{D}$ has better convergence performance. Consequently, we demonstrate how $\mathrm{AnD}$ is able to strike a better balance between convergence and diversity than the five competitors. 


\subsection{Computational Time Complexity}

The computational time complexity of $\mathrm{AnD}$ is dependent mainly on its environmental selection. In Algorithm 2, since the vector angles should be computed between any two individuals in the union population $\mathcal{U}_{t}$ of size $2 N$, the computational time complexity is thus $O\left(m N^{2}\right)$. In addition, the computational time complexity of sorting these vector angles is $O\left(N^{2} \log _{2} N\right)$. The implementation of the shift-based density estimation strategy has a time complexity of $O\left(m N^{2}\right)$. Therefore, the overall computational time complexity of $\mathrm{AnD}$ at one generation is $\max \left\{O\left(m N^{2}\right), O\left(N^{2} \log _{2} N\right)\right\}$.

\subsection{Discussion}

AnD abandons the use of dominance rules, weight vectors or reference points, and indicators. As a result, AnD alleviates the disadvantages of other MaOEAs to some extent when solving MaOPs, such as the loss of selection pressure in Pareto-based approaches, the requirement of specifying weight vectors or reference points in decomposition-based methods, and the high computational time complexity in hypervolume-based approaches. AnD also has some other good properties. For instance, it has a simple structure, few parameters, and no complicated operators. Actually, it is an effectiye algorithm for solving both unconstrained and constrained MaOPs as demonstrated in Section 5.

As introduced in Section 2, RVEA [4], MOEA/D-LWS [39], MOEA/D-SAS [3], VaEA [40], and MaOEA-CSS [15] utilize the information of the vector angle, while SPEA2+SDE [27], CMODE+SDE [36], and SRA [24] employ shift-based density estimation. To the best of our knowledge, AnD is the first attempt to combine both the vector angle with the shift-based density estimation strategy, by taking advantage of their complementary features.

\section{Experimental Setup}

\subsection{Benchmark Test Problems}

To evaluate the performance of the proposed AnD, we applied it to solve two well-known benchmark test suites, namely, the DTLZ [10] and WFG [17] test suites. DTLZ1-DTLZ4 and WFG1-WFG9 with five, 10, and 15 objectives were chosen for our empirical studies. Following the suggestion in [10], the number of decision variables $n$ was set to $n=m+k-1$ for the DTLZ test suite, where $m$ denotes the number of objectives, $k=5$ for DTLZ1, and $k=10$ for DTLZ2DTLZ4. As recommended in [17], $n$ was set to $n=k+l$ for the WFG test suite, 
where the position-related variable $k=2 \times(m-1)$, and the distance-related variable $l=20$.

As pointed out in [10] and [17], the PFs of the DTLZ and WFG test suites have various characteristics (i.e., linear, convex, concave, mixed, and multi-modal), which pose a great challenge for a MaOEA to find a well-converged and welldistributed solution set.

\subsection{Performance Metrics}

Two widely used performance metrics—inverted generational distance(IGD) [5] and hypervolume (HV) [47]—were employed to compare AnD with other MaOEAs.

- IGD: Suppose that $\mathcal{P}$ is an approximation set, and $\mathcal{P}^{*}$ is a set of nondominated solutions uniformly distributed on the true PF. The IGD metric is calculated as:

$$
I G D(\mathcal{P})=\frac{1}{\left|\mathcal{P}^{*}\right|} \sum_{\mathbf{z}^{*} \in \mathcal{P}^{*}} \text { distance }\left(\mathbf{z}^{*}, \mathcal{P}\right) .
$$

where distance $\left(\mathbf{z}^{*}, \mathcal{P}\right)$ is the minimum Euclidean distance between $\mathbf{z}^{*}$ and all members in $\mathcal{P}$, and $\left|\mathcal{P}^{*}\right|$ is the cardinality of $\mathcal{P}^{*}$. The smaller the IGD value, the better the performance of a MaOEA.

- $H V$ : HV measures the volume enclosed by $\mathcal{P}$ and a specified reference point in the objective space [12]. It assesses both the convergence and diversity of $\mathcal{P}$, and is the only indicator which is Pareto-compliant [2]. For a MaOEA, a larger $\mathrm{HV}$ value is desirable. In our experiments, firstly, the objective vectors of $\mathcal{P}$ are normalized. Thereafter, the $\mathrm{HV}$ value is calculated by using the reference point which is set to 1.1 times the upper bounds of the true PF. To approximate the exact HV value, the Monte Carlo sampling [2] is usually adopted.

\subsection{Algorithms for Comparison}

The following seven state-of-the-art MaOEAs are under our consideration for performance comparison.

- RVEA [4]: RVEA is a reference-vector-guided EA for many-objective optimization. In RVEA, the angle between the reference vector and the objective vector is used to compute the angle-penalized distance. 
Table 1: Population size of three algorithms

\begin{tabular}{cccc}
\hline$m$ & No. of Vectors & NSGA-III & MOEA/D and MOEA/DD \\
\hline 5 & 210 & 212 & 210 \\
10 & 275 & 276 & 275 \\
15 & 135 & 136 & 135 \\
\hline
\end{tabular}

- SPEA2+SDE [27]: SPEA2+SDE incorporates shift-based density estimation into SPEA2 for MaOPs.

- MOEA/D [42]: Herein, MOEA/D with the penalty-based boundary intersection (PBI) function is used in our experiments.

- NSGA-III [8]: NSGA-III is a reference-point-based MaOEA following the NSGA-II framework.

- MOMBI-II [16]: MOMBI-II is a recently proposed indicator-based MaOEA, which adopts the $R 2$ indicator as the selection criterion.

- MOEA/DD [26]: MOEA/DD is based on both Pareto dominance and decomposition.

- Two_Arch2 [35]: Two_Arch2 assigns different selection principles (indicatorbased and Pareto-based) to two archives for convergence and diversity, respectively.

\subsection{Parameter Settings}

- Population Size: Table 1 presents the population size of NSGA-III, MOEA/D, and MOEA/DD. For other algorithms, the population size was kept the same as NSGA-III.

- Parameter Settings for Evolutionary Operators: For all algorithms, SBX and the polynomial mutation were used to produce offspring. The crossover probability and the mutation probability were set to 1.0 and $1 / n$, respectively, and the distribution indexes of both SBX and the polynomial mutation were set to 20 .

- Number of Independent Runs and Termination Condition: All algorithms were independently run 20 times on each test problem, and terminated when 90,000 function evaluations (FEs) were reached [35, 24]. 
- Parameter Settings for Algorithms: For RVEA [4], $\alpha=2$ and $f_{r}=0.1$ for all test problems following the suggestion in [4]. For MOEA/D [42], the neighborhood size was set to 20 , the maximum replacement number was set to 2 , and the penalty parameter $\theta$ was set to 5. For MOEA/DD [26], the neighborhood size and $\theta$ were kept the same as MOEA/D, and the probability $\delta$ was set to 0.9 . According to [16], two parameters in MOMBI-II were set as $\epsilon=0.001$ and $\alpha=0.5$, respectively.

In this paper, all the experiments were implemented in the platform recently developed by Tian et al. [31].

\section{Results and Discussions}

\subsection{Benefit of Two Strategies}

Firstly, we are interested in identifying the benefit of two crucial strategies of AnD: angle-based selection and shift-based density estimation. To this end, two variants of $\mathrm{AnD}$ were devised named AnD-WoA and AnD-WoD, respectively. In AnD-WoA, angle-based selection was eliminated. Instead, the individuals in the union population $\mathcal{U}_{t}$ were sorted based on their shift-based density values [25], and $N$ individuals with the highest density values were removed from $\mathcal{U}_{t}$. In AnD-WoD, shift-based density estimation was abandoned. As an alternative, for two individuals with the minimum vector angle, their Euclidean distances to the ideal point were computed, and the one with the larger Euclidean distance was deleted $[11,15]$. The comparative experiments between $\mathrm{AnD}$ and its two variants were carried out on the DTLZ and WFG test suites. The IGD and HV values are shown in Table 2 and Table 3 , respectively.

From Table 2 and Table 3, it is evident that AnD outperforms its two variants on a vast majority of test problems. In terms of the IGD metric, AnD obtains the best performance on 30 out of 39 test problems, while AnD-WoA and AnDWoD achieve the best performance on only five and four test problems, respectively. With respect to the HV metric, AnD performs the best on 36 test problems. Nevertheless, AnD-WoA and AnD-WoD perform the best on no more than two test problems. The reason for the above results seems obvious. Compared with AnD, AnD-WoA discards angle-based selection, therefore it is unable to maintain the diversity of search directions. Regarding AnD-WoD, it replaces shift-based density estimation with the Euclidean distance. However, the Euclidean distance only considers an individual's convergence property; thus, it is less reasonable 
Table 2: Performance comparison of AnD, AnD-WoA, and AnD-WoD in terms of the average IGD value on the DTLZ and WFG test suites. The best average IGD value among all the algorithms on each test problem is highlighted in Gray.

\begin{tabular}{|c|c|c|c|c|}
\hline Problem & $m$ & AnD-WoA & AnD-WoD & $\mathrm{AnD}$ \\
\hline DTLZ1 & 5 & $7.1041 e-2$ & $7.3325 e-2$ & $6.0100 e-2$ \\
\hline DTLZ1 & 10 & $1.2443 e-1$ & $1.6631 e-1$ & $1.2504 e-1$ \\
\hline DTLZ1 & 15 & $1.7912 e-1$ & $2.3414 e-1$ & $1.9064 e-1$ \\
\hline DTLZ2 & 5 & $2.4507 e-1$ & $1.6893 e-1$ & $1.6826 e-1$ \\
\hline DTLZ2 & 10 & $4.8020 e-1$ & $4.5503 e-1$ & $3.7456 e-1$ \\
\hline DTLZ2 & 15 & $7.4842 e-1$ & $7.3188 e-1$ & $5.4876 e-1$ \\
\hline DTLZ3 & 5 & $2.5380 e-1$ & $1.9300 e-1$ & $1.8791 e-1$ \\
\hline DTLZ3 & 10 & $4.8583 e-1$ & $4.9184 e-1$ & $1.1336 e+0$ \\
\hline DTLZ3 & 15 & $7.6376 e-1$ & $9.1091 e-1$ & $1.8929 e+0$ \\
\hline DTLZ4 & 5 & $2.4661 e-1$ & $1.6692 e$ & $1.6868 e-1$ \\
\hline DTLZ4 & 10 & $4.5454 e-1$ & $4.0055 e-1$ & $3.7863 e-1$ \\
\hline DTLZ4 & 15 & $6.8081 e-1$ & $5.9132 e-1$ & $5.5382 e-1$ \\
\hline WFG1 & 5 & $9.3495 e-1$ & $1.0391 e+0$ & $8.2485 e-1$ \\
\hline WFG1 & 10 & $1.9120 e+0$ & $2.3222 e+0$ & $1.8344 e+0$ \\
\hline WFG1 & 15 & $4.2708 e+0$ & $4.4120 e+0$ & $2.5826 e+0$ \\
\hline WFG2 & 5 & $1.2924 e+0$ & $1.2482 e+0$ & $7.4199 e-1$ \\
\hline WFG2 & 10 & $4.8891 e+0$ & $4.6090 e+0$ & $3.7346 e+0$ \\
\hline WFG2 & 15 & $1.4048 e+1$ & $1.4005 e+1$ & $1.2394 e+1$ \\
\hline $\begin{array}{l}-\cdots-\cdots \\
\text { WFG3 }\end{array}$ & 5 & $1.0499 e+0$ & $8.2442 e-1$ & $5.0305 e-1$ \\
\hline WFG3 & 10 & $1.5811 e+0$ & $2.1080 e+0$ & $1.7025 e+0$ \\
\hline WFG3 & 15 & $8.6690 e+0$ & $1.2946 e+0$ & $2.6156 e+0$ \\
\hline WF & 5 & $1.2669 e+0$ & $9.4798 e-1$ & $9.5061 e-1$ \\
\hline WFG4 & 10 & $4.6742 e+0$ & $4.3908 e+0$ & $3.6441 e+0$ \\
\hline WRC & 15 & $1.0007 e+1$ & $1.0596 e+1$ & $7.6264 e+0$ \\
\hline WFG5 & 5 & $1.2722 e+0$ & $9.5546 e-1$ & $9.3925 e-1$ \\
\hline WFG5 & 10 & $4.7402 e+0$ & $4.2061 e+0$ & $3.5788 e+0$ \\
\hline WFG5 & 15 & $1.0252 e+1$ & $9.4419 e+0$ & $7.5925 e+0$ \\
\hline WFG6 & 5 & $1.3676 e+0$ & $9.3884 e-1$ & $9.5995 e-1$ \\
\hline WFG6 & 10 & $4.7496 e+0$ & $4.0846 e+0$ & $3.5574 e+0$ \\
\hline WFG6 & 15 & $1.1015 e+1$ & $1.0492 e+1$ & $7.5193 e+0$ \\
\hline WFG7 & 5 & $13386 e+0$ & $957170-1$ & $9.5631 e-1$ \\
\hline WFG7 & 10 & $4.6176 e+0$ & $4.1528 e+0$ & $3.4909 e+0$ \\
\hline WFG7 & 15 & $1.0715 e+1$ & $9.7741 e+0$ & $7.5817 e+0$ \\
\hline WFG8 & 5 & $1.3795 e+0$ & $1.0943 e+0$ & $1.0138 e+0$ \\
\hline WFG8 & 10 & $5.4254 e+0$ & $5.0135 e+0$ & $3.8497 e+0$ \\
\hline WFG8 & 15 & $1.1483 e+1$ & $1.2461 e+1$ & $8.8015 e+0$ \\
\hline WFG9 & 5 & $1.2789 e+0$ & $1.0351 e+0$ & $9.4961 e-1$ \\
\hline WFG9 & 10 & $4.9261 e+0$ & $4.6810 e+0$ & $3.9489 e+0$ \\
\hline WFG9 & 15 & $1.0603 e+1$ & $9.8005 e+0$ & $8.0252 e+0$ \\
\hline
\end{tabular}


Table 3: Performance comparison of of AnD, AnD-WoA, and AnD-WoD in terms of the average HV value on the DTLZ and WFG test suites. The best average HV value among all the algorithms on each test problem is highlighted in Gray.

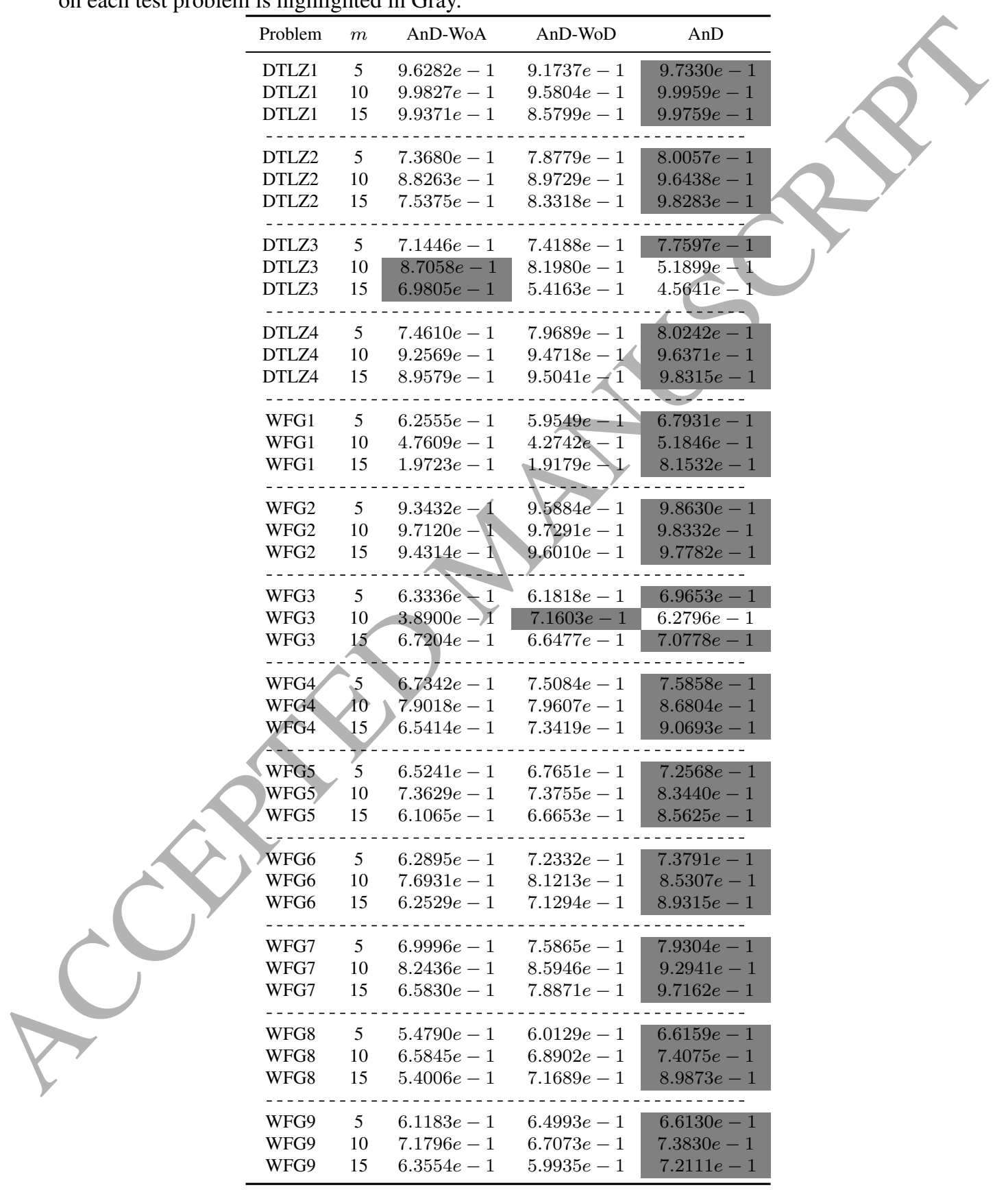


Table 4: Performance comparison between AnD and seven state-of-the-art MaOEAs in terms of the average IGD value on the DTLZ test suite. The best and second best average IGD values among all the algorithms on each test problem are highlighted in Gray and light Gray, respectively.

\begin{tabular}{|c|c|c|c|c|c|c|c|c|c|}
\hline Problem & $m$ & RVEA & SPEA2+SDE & MOEA/D & NSGA-III & MOMBI-II & MOEA/DD & Two_Arch2 & $\mathrm{AnD}$ \\
\hline DTLZ1 & 5 & $5.2408 e-2$ & $5.0463 e-2$ & $5.2640 e-2$ & $5.2550 e-2$ & $5.2675 e-2$ & $5.2435 e-2$ & $5.3565 e-2$ & $6.0100 e-$ \\
\hline DTLZ1 & 10 & $1.4004 e-1$ & $1.1292 e-1$ & $1.1831 e-1$ & $1.8856 e-1$ & $2.1156 e-1$ & $1.2387 e-1$ & $1.2356 e-1$ & $1.2504 e-1$ \\
\hline DTLZ1 & 15 & $1.8157 e-1$ & $1.6530 e-1$ & $1.6486 e-1$ & $2.6475 e-1$ & $3.1515 e-1$ & $1.6710 e-1$ & $1.8887 e-1$ & $1.9064 e-1$ \\
\hline DTLZ2 & 5 & $1.6124 e-1$ & $1.8868 e-1$ & $1.6124 e-1$ & $1.6125 e-1$ & $1.6307 e-1$ & $1.6124 e-$ & $1.7374 e$ & $1.6826 e-1$ \\
\hline DTLZ2 & 10 & $4.1870 e-1$ & $3.8855 e-1$ & $4.2106 e-1$ & $4.9906 e-1$ & $4.1920 e-1$ & $4.2108 e-1$ & $4.5879 e-1$ & $3.7456 e-1$ \\
\hline DTLZ2 & 15 & $5.7768 e-1$ & $5.5854 e-1$ & $5.7862 e-1$ & $6.4743 e-1$ & $8.3078 e-1$ & $5.7824 e-1$ & $6.7923 e-1$ & $5.4876 e-1$ \\
\hline DTLZ3 & 5 & $1.7390 e-1$ & $1.8818 e-1$ & $1.6662 e-1$ & $1.7486 e-1$ & $1.7057 e-1$ & $1.6305 e-$ & $2 e-$ & $1.8791 e-1$ \\
\hline DTLZ3 & 10 & $6.4615 e-1$ & $3.9359 e-1$ & $8.3022 e-1$ & $9.5607 e+0$ & $4.8416 e-1$ & $4.5511 e-1$ & $1.8047 e+0$ & $1.1336 e+0$ \\
\hline DTLZ3 & 15 & $7.3919 e-1$ & $5.7993 e-1$ & $1.1593 e+0$ & $3.1400 e+1$ & $1.1082 e+0$ & $5.9058 e-1$ & $6.6586 e+0$ & $1.8929 e+0$ \\
\hline DTLZ4 & 5 & $1.6122 e-1$ & $1.8854 e-1$ & $4.6392 e-1$ & $1.6128 e-1$ & $1.6474 e-1$ & $1.6122 e-$ & $1.7605 e-1$ & $1.6868 e-1$ \\
\hline DTLZ4 & 10 & $4.1616 e-1$ & $3.9027 e-1$ & $6.2527 e-1$ & $4.4139 e-1$ & $4.2156 e-1$ & $4.2107 e-$ & $4.5855 e-1$ & $3.7863 e-1$ \\
\hline DTLZ4 & 15 & $5.8259 e-1$ & $5.5802 e-1$ & $7.7231 e-1$ & $6.2785 e-1$ & $5.9901 e-1$ & $5.8698 e-1$ & $6.7621 e-1$ & $5.5382 e-1$ \\
\hline
\end{tabular}
the average HV value on the DTLZ test suite. The best and second best average HV values among all the algorithms on each test problem are highlighted in Gray and light Gray, respectively.

\begin{tabular}{|c|c|c|c|c|c|c|c|c|c|}
\hline Problem & $m$ & RVEA & SPEA2+SDE & MOEA/D & NSGA-III & MOMBI-II & MOEA/DD & Two_Arch2 & $\mathrm{AnD}$ \\
\hline DTLZ1 & 5 & $9.7971 e-1$ & $9.6924 e-1$ & $9.7942 e-1$ & $9.7962 e$ & $9.7942 e-1$ & $9.7977 e-1$ & $9.7582 e-1$ & $9.7330 e-1$ \\
\hline DTLZ1 & 10 & $9.9868 e-1$ & $9.9598 e-1$ & $9.9826 e-1$ & $9.1305 e$ & $9.6719 e-1$ & $9.9962 e-1$ & $9.9507 e-1$ & $9.9894 e-1$ \\
\hline DTLZ1 & 15 & $9.9950 e-1$ & $9.9122 e-1$ & $9.5880 e-1$ & $9.0076 e-$ & $8.2655 e-1$ & $9.9707 e-1$ & $9.8287 e-1$ & $9.9759 e-1$ \\
\hline DTLZ2 & 5 & $8.1234 e-1$ & $8.1125 e-1$ & $8.1254 e-1$ & $8.1233 e$ & $8.1174 e-1$ & $8.1247 e-1$ & $7.6937 e-1$ & $8.0057 e-1$ \\
\hline DTLZ2 & 10 & $9.7440 e-1$ & $9.7222 e-1$ & $9.7529 e-1$ & $9.2862 e$ & $9.7439 e-1$ & $9.7531 e-1$ & $7.7695 e-1$ & $9.6438 e-1$ \\
\hline DTLZ2 & 15 & $9.8964 e-1$ & $9.8403 e-1$ & $9.8841 e-1$ & $9.3018 e$ & $8.4042 e-1$ & $9.9015 e-1$ & $6.3825 e-1$ & $9.8283 e-1$ \\
\hline DTLZ3 & 5 & $7.7880 e-1$ & $8.0874 e$ & $7.8499 e-1$ & 7,7721 & $8.0386 e-1$ & $7.9779 e-1$ & $7.2701 e-1$ & $7.7597 e-1$ \\
\hline DTLZ3 & 10 & $7.0475 e-1$ & $9.6885 e-1$ & $4.9755 e-1$ & $0.0000 e+0$ & $9.3247 e-1$ & $9.2789 e-1$ & $1.6481 e-1$ & $5.1899 e-1$ \\
\hline DTLZ3 & 15 & $7.6387 e-1$ & $9.7532 e-1$ & $2.3293 e-1$ & $0.0000 e+0$ & $4.3883 e-1$ & $9.7556 e-1$ & $0.0000 e+0$ & $4.5641 e-1$ \\
\hline DTLZ4 & 5 & $8.1251 e-1$ & 8.1225 & -1 & $8.1186 e-1$ & $8.1128 e-1$ & $8.1256 e-1$ & $7.5774 e-1$ & $8.0242 e-1$ \\
\hline DTLZ4 & 10 & $9.7383 e-1$ & $9.7124 e$ & $8.7615 e-1$ & $9.6258 e-1$ & $9.7453 e-1$ & $9.7536 e-1$ & $7.8132 e-1$ & $9.6371 e-1$ \\
\hline DTLZ4 & 15 & $9.8789 e-1$ & $9.8745 e-$ & $8.8487 e-1$ & $9.5702 e-1$ & $9.8474 e-1$ & $9.8837 e-1$ & $6.3605 e-1$ & $9.8315 e-1$ \\
\hline
\end{tabular}

than shift-based density estimation which takes both diversity and convergence into account.

From this discussion, we can conclude that angle-based selection and shiftbased density estimation are two indispensable strategies in AnD.

\subsection{Comparison with Seven State-of-the-Art MaOEAs}

We compared the performance of AnD with that of the seven peer algorithms introduced in Section 4.3 on the DTLZ and WFG test suites in terms of the IGD and HV metrics. The results are summarized in Tables 4-7. At first glance, RVEA, SPEA2+SDE, and MOEA/DD achieve superior performance on the DTLZ test suite. The reason might be that the DTLZ test suite puts more emphasis on an algorithm's convergence ability than its ability to diversity [20]. Note, however, that 


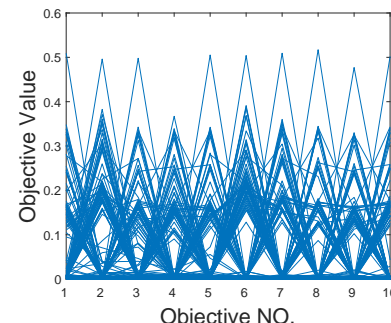

(a) RVEA

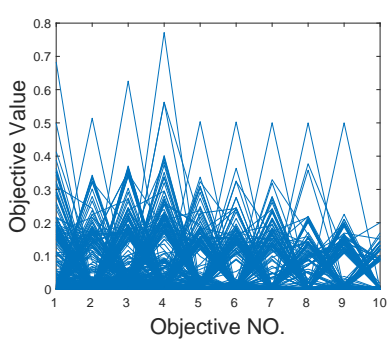

(d) NSGA-III

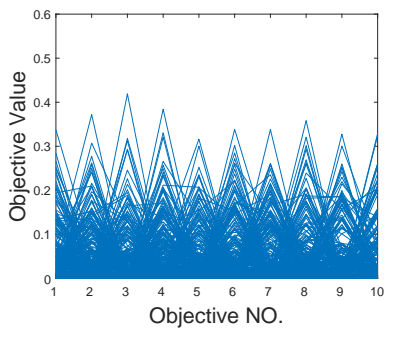

(b) SPEA2+SDE

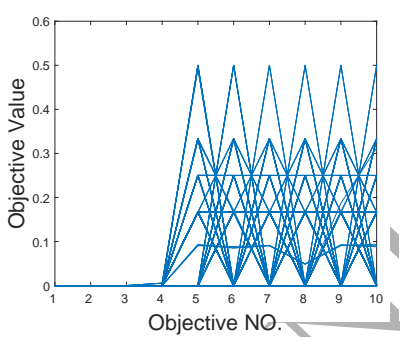

(e) MOMBI-II

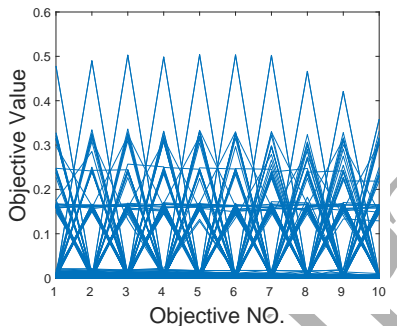

(c) MOEA/D

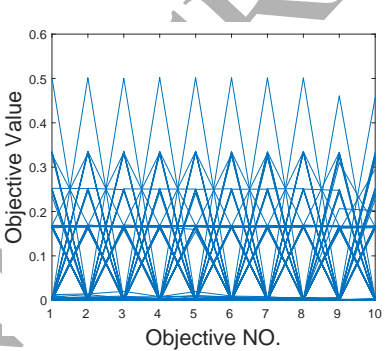

(f) MOEA/DD

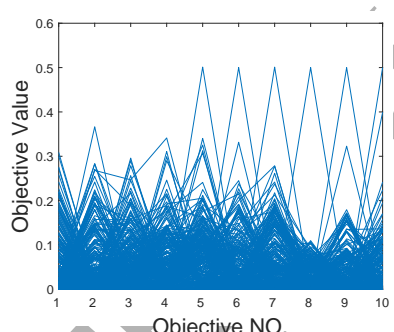

(g) Two_Arch2

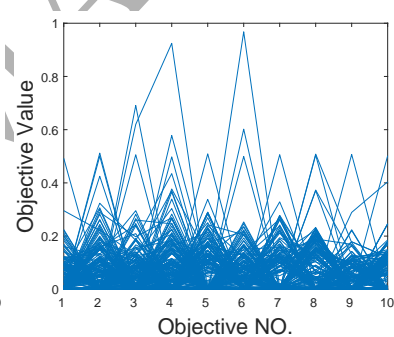

(h) $\mathrm{AnD}$

Figure 4: The final solution sets of the eight compared algorithms on DTLZ1 with ten objectives by parallel coordinates.

AnD obtains the best overall performance on the WFG test suite. To visualize the results, we plotted the final populations resulting from the eight compared algorithms in a typical run by parallel coordinates on four representative test problems in Figs. 4-7. Note that a typical run means a run producing the median IGD value among all runs. The detailed discussions are given next. 


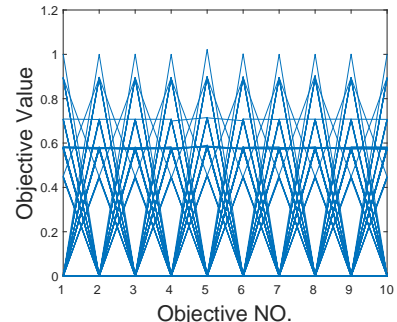

(a) RVEA

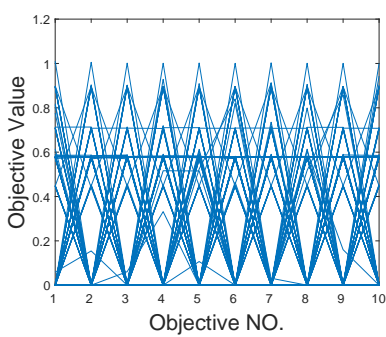

(d) NSGA-III

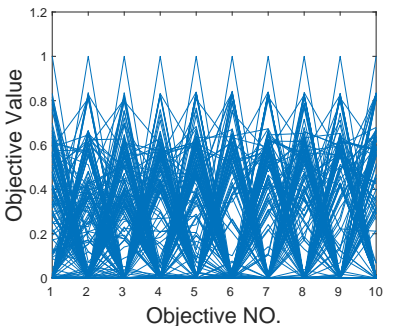

(b) SPEA2+SDE

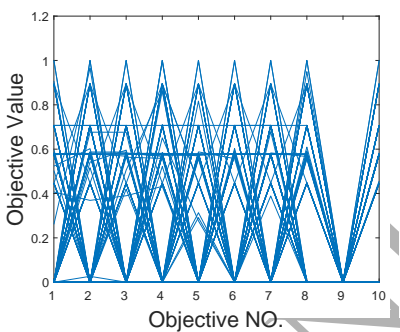

(e) MOMBI-II

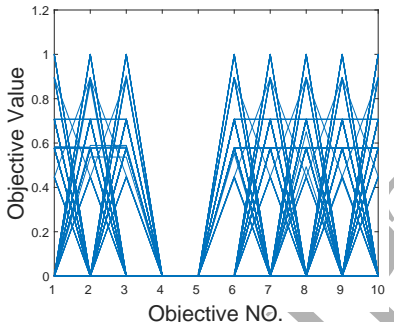

(c) MOEA/D

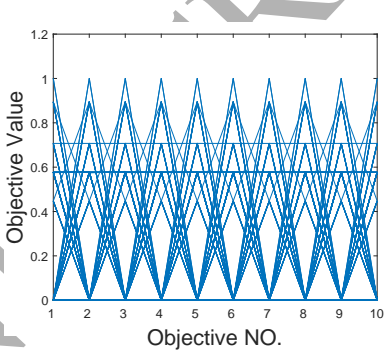

(f) MOEA/DD

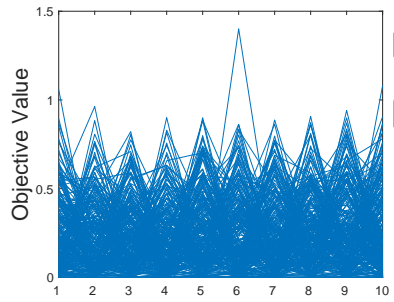

Objective NO

(g) Two_Arch2

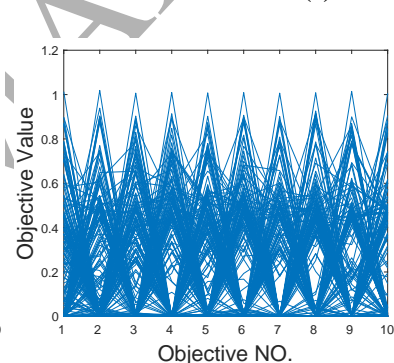

(h) $\mathrm{AnD}$

Figure 5: The final solution sets of the eight compared algorithms on DTLZ4 with ten objectives by parallel coordinates.

\subsubsection{DTLZ Test Suite}

From Tables 4 and 5, we observe that the eight compared algorithms exhibit mixed performance. More specifically, SPEA2+SDE performs the best in terms of the IGD metric, followed by MOEA/DD and AnD, as shown in Table 4. Nevertheless, MOEA/DD and RVEA obtain the best and second best performance with respect to the HV metric, respectively, as shown in Table 5. 
DTLZ1 is a multimodal problem, in which the PF is degenerate and the decision variables are non-separable. It challenges the convergence performance of an algorithm. We find that SPEA2+SDE achieves the best IGD values on five and 10 objectives, while MOEA/D obtains the best IGD value on 15 objectives. As far as HV is concerned, MOEA/DD outperforms the others on five and 10 objectives, and RVEA beats its competitors on 15 objectives. As shown in Fig. 4, the results provided by some methods using weight vectors or reference points (i.e., MOEA/D and MOEA/DD) have better distributions. With respect to SPEA2+SDE and Two_Arch2, the scales of some objective values are smaller than the true PF, which suggests that they have a preference on the solutions located in central areas. In terms of $\mathrm{AnD}$, some extreme values for the third, fourth, and sixth objectives occur, which means that $\mathrm{AnD}$ has relatively poor convergence performance on DTLZ1.

DTLZ2 is a relatively simple test problem compared with DTLZ1, which is mainly used to test an algorithm's diversity. AnD achieves the best overall IGD performance, while MOEA/DD obtains the best overall HV performance. In $\mathrm{AnD}$, the diversity of the population is considered in both angle-based selection and shift-based density estimation; therefore, $\mathrm{AnD}$ provides promising results on DTLZ2.

For DTLZ3, which is a highly multimodal problem, SPEA2+SDE achieves the best overall performance in terms of both IGD and HV. From Table 5, it can be seen that the results provided by NSGA-III and Two_Arch2 are distant from the PFs on 10 and 15 objectives. AnD obtains medium performance in terms of both IGD and HV.

Regarding DTLZ4, the density of points on its PF is strongly biased. Therefore, the main challenge for solving this test problem is to maintain the diversity of the population. Similar to DTLZ2, AnD and MOEA/DD obtain the best overall IGD and HV performance, respectively. From Fig. 5, it can be observed that Two_Arch 2 produces some extreme values on the sixth objective, which suggests unstable convergence performance. The results obtained by RVEA, NSGA-III, and MOEA/DD distribute similarly and concentrate mainly on the boundary or the middle parts of the PF. As for MOEA/D, its results fail to cover the fourth and fifth objectives well. Similarly, the results obtained by MOMBI-II are unable to cover the ninth objective well. SPEA2+SDE and AnD are capable of covering the whole PF. The difference between them is that the objective values derived from SPEA2+SDE mainly lie in $[0,0.8]$, while in $\mathrm{AnD}$, they are well distributed within $[0,1]$. 
Table 6: Performance comparison between AnD and seven state-of-the-art MaOEAs in terms of the average IGD value on the WFG test suite. The best and second best average IGD values among all the algorithms on each test problem are highlighted in Gray and light Gray, respectively.

\begin{tabular}{|c|c|c|c|c|c|c|c|c|c|}
\hline Problem & $m$ & RVEA & SPEA2+SDE & MOEA/D & NSGA-III & MOMBI-II & MOEA/DD & Two_Arch2 & $\mathrm{AnD}$ \\
\hline WFG1 & 5 & $1.2380 e+0$ & $4.5051 e-1$ & $1.4719 e+0$ & $1.3003 e+0$ & $7.3247 e-1$ & $1.7863 e+0$ & $9.5226 e-1$ & $8.2485 e$ \\
\hline WFG1 & 10 & $2.4159 e+0$ & $1.5483 e+0$ & $3.5093 e+0$ & $2.3319 e+0$ & $2.9849 e+0$ & $2.7158 e+0$ & $2.2678 e+0$ & $1.8344 e+0$ \\
\hline WFG1 & 15 & $3.4128 e+0$ & $3.0000 e+0$ & $5.4507 e+0$ & $3.6069 e+0$ & $6.0947 e+0$ & $4.2376 e+0$ & $3.1632 e+0$ & $2.5826 e+0$ \\
\hline WFG2 & 5 & $7.3061 e-1$ & $9.9429 e-1$ & $2.9028 e+0$ & $6.2450 e-1$ & $1.2746 e+0$ & $1.9358 e+0$ & $4.7796 e$ & 1 \\
\hline WFG2 & 10 & $3.6478 e+0$ & $5.4046 e+0$ & $1.1021 e+1$ & $4.0066 e+0$ & $3.9907 e+0$ & $1.1009 e+1$ & $1.9916 e+0$ & $3.7346 e+0$ \\
\hline WFG2 & 15 & $1.2716 e+1$ & $1.4549 e+1$ & $1.6855 e+1$ & $1.2405 e+1$ & $1.9653 e+1$ & $1.5737 e+1$ & $5.7470 e+0$ & $1.2394 e+1$ \\
\hline WFG3 & 5 & $6.4564 e-1$ & $5.8114 e-1$ & $1.0504 e+0$ & $4.7212 e-1$ & $9.5483 e-1$ & $6.5805 e-1$ & 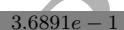 & $5.0305 e-1$ \\
\hline WFG3 & 10 & $3.0551 e+0$ & $1.7028 e+0$ & $8.6850 e+0$ & $8.7259 e-1$ & $9.3633 e+0$ & $2.8279 e+0$ & $1.2887 e+0$ & $1.7025 e+0$ \\
\hline WFG3 & 15 & $6.2924 e+0$ & $4.5129 e+0$ & $1.6375 e+1$ & $3.2111 e+0$ & $1.6002 e+1$ & $1.4214 e+1$ & $2.5444 e+0$ & $2.6156 e+0$ \\
\hline WFG4 & 5 & $9.4475 e-1$ & $1.1148 e+0$ & $1.6793 e+0$ & $9.5187 e-1$ & $1.7483 e+0$ & $1.0314 e+0$ & $9.7618 e-1$ & $9.5061 e-1$ \\
\hline WFG4 & 10 & $3.8034 e+0$ & $4.1323 e+0$ & $8.9303 e+0$ & $4.0807 e+0$ & $8.3168 e+0$ & $5.2137 e+0$ & $4.5821 e+0$ & $3.6441 e+0$ \\
\hline WFG4 & 15 & $8.7523 e+0$ & $8.2858 e+0$ & $1.6011 e+1$ & $8.9661 e+0$ & $2.3009 e+1$ & $9.1948 e+0$ & $9.7013 e+0$ & $7.6264 e+0$ \\
\hline WFG5 & 5 & $9.3668 e-1$ & $1.1233 e+0$ & $1.5112 e+0$ & $9.3688 e-1$ & 1.6 & 0 & $9.7161 e-1$ & $9.3925 e-1$ \\
\hline WFG5 & 10 & $3.8534 e+0$ & $4.0242 e+0$ & $8.6325 e+0$ & $3.8863 e+0$ & $7.2474 e+0$ & $5.9057 e+0$ & $4.5803 e+0$ & $3.5788 e+0$ \\
\hline WFG5 & 15 & $8.2300 e+0$ & $1.0363 e+1$ & $1.6180 e+1$ & $8.6808 e+0$ & $2.7050 e+1$ & $1.3822 e+1$ & $9.6726 e+0$ & $7.5925 e+0$ \\
\hline WFG6 & 5 & $9.4927 e-1$ & $1.1683 e+04$ & $1.9082 e+0$ & $9.5167 e-1$ & 1.6161 & $0294 e+0$ & $9.8289 e-1$ & $9.5995 e-1$ \\
\hline WFG6 & 10 & $3.8553 e+0$ & $4.1209 e+0$ & $9.7139 e+0$ & $3.9288 e+0$ & $7.5219 e+0$ & $5.4913 e+0$ & $4.5845 e+0$ & $3.5574 e+0$ \\
\hline WFG6 & 15 & $8.5768 e+0$ & $9.3893 e+0$ & $1.6228 e+1$ & $9.0585 e+0$ & $2.3092 e+1$ & $1.3704 e+1$ & $9.7084 e+0$ & $7.5193 e+0$ \\
\hline WFG7 & 5 & $9.5376 e-1$ & $1.1481 e+0$ & $1.7551 e+0$ & $9.5434 e-$ & $1.4084 e+0$ & $1.0384 e+0$ & $9.5596 e-1$ & $9.5631 e-1$ \\
\hline WFG7 & 10 & $3.8105 e+0$ & $3.9180 e+0$ & $9.6364 e+0$ & $4.1474 e+0$ & $7.0575 e+0$ & $4.8005 e+0$ & $4.5546 e+0$ & $3.4909 e+0$ \\
\hline WFG7 & 15 & $8.4477 e+0$ & $8.1509 e+0$ & $1.6400 e+1$ & $9.2815 e+0$ & $2.2087 e+1$ & $8.0829 e+0$ & $9.9669 e+0$ & $7.5817 e+0$ \\
\hline WFG8 & 5 & $9.8533 e-1$ & $1.1560 e+0$ & $1.2965 e+0$ & $1.0084 e+0$ & $2.3995 e+0$ & $1.0543 e+0$ & $1.1102 e+0$ & $1.0138 e+0$ \\
\hline WFG8 & 10 & $3.9040 e+0$ & $4.3343 e+0$ & $8.1439 e+0$ & $5.1141 e+0$ & $8.8767 e+0$ & $4.1808 e+0$ & $5.4467 e+0$ & $3.8497 e+0$ \\
\hline WFG8 & 15 & $9.5526 e+0$ & $8.5588 e+0$ & $1.2660 e+1$ & $1.0520 e+1$ & $2.3945 e+1$ & $9.0171 e+0$ & $1.0866 e+1$ & $8.8015 e+0$ \\
\hline WFG9 & 5 & $9.1032 e-1$ & $1.0663 e+0$ & $1.3802 e+0$ & $9.2301 e-1$ & $2.2015 e+0$ & $1.0173 e+0$ & $1.0066 e+0$ & $9.4961 e-1$ \\
\hline WFG9 & 10 & $4.1319 e+0$ & $4.2572 e+0$ & $9.0024 e+0$ & $4.1250 e+0$ & $6.7789 e+0$ & $6.0132 e+0$ & $5.1443 e+0$ & $3.9489 e+0$ \\
\hline WFG9 & 15 & $8.2901 e+0$ & $9.1482 e+0$ & $1.5257 e+1$ & $8.6989 e+0$ & $2.7200 e+1$ & $1.2606 e+1$ & $1.0647 e+1$ & $8.0252 e+0$ \\
\hline
\end{tabular}

\subsubsection{WFG Test Suite}

The first observation from Tables 6 and 7 is that $\mathrm{AnD}$ attains the best overall performance in terms of both IGD and HV. Next, we give the detailed discussions.

Table 6 shows the IGD values resulting from the eight compared algorithms. Clearly, AnD and RVEA are the two top algorithms, and they have a clear advantage over the other six algorithms on the majority of test problems. Actually, AnD provides the best and second best IGD values on 12 and six out of 27 test problems, respectively. As for RVEA, it generates six best results and nine second best results. In addition, SPEA2+SDE obtains three best results and two second best results, Two_Arch2 produces five best results, NSGA-III has one best result and seven second best results, and MOEA/DD and MOMBI-II reach one second best result each. One interesting phenomenon we have observed is that the methods based on weight vectors or reference points (i.e., MOEA/D, MOEA/DD, NSGA-III, and MOMBI-II) seem to lose their effectiveness on this test suite. This 
Table 7: Performance comparison between AnD and seven state-of-the-art MaOEAs in terms of the average HV value on the WFG test suite. The best and second best average HV values among all the algorithms on each test problem are highlighted in Gray and light Gray, respectively.

\begin{tabular}{|c|c|c|c|c|c|c|c|c|c|}
\hline Problem & $m$ & RVEA & SPEA2+SDE & MOEA/D & NSGA-III & MOMBI-II & MOEA/DD & Two_Arch2 & AnD \\
\hline WFG1 & 5 & $5.2614 e-1$ & $8.5804 e-1$ & $6.9318 e-1$ & $5.0964 e-1$ & $9.8249 e-1$ & $3.7722 e-1$ & $6.3042 e-1$ & $6.7931 e$ \\
\hline WFG1 & 10 & $3.3523 e-1$ & $6.1873 e-1$ & $4.5187 e-1$ & $4.2400 e-1$ & $9.9177 e-1$ & $2.8866 e-1$ & $3.9733 e-1$ & $5.1846 e-$ \\
\hline WFG1 & 15 & $6.4838 e-1$ & $6.4360 e-1$ & $2.5320 e-1$ & $5.6188 e-1$ & $8.3535 e-1$ & $8.5739 e-1$ & $4.8177 e-1$ & $8.1532 e-1$ \\
\hline WFG2 & 5 & $9.5415 e-1$ & $9.4272 e-1$ & $7.4322 e-1$ & $9.6190 e-1$ & $9.7075 e-1$ & $9.2248 e-1$ & $9.7553 e-1$ & \\
\hline WFG2 & 10 & $9.0610 e-1$ & $9.5588 e-1$ & $7.3343 e-1$ & $9.4637 e-1$ & $7.5468 e-1$ & $8.8639 e-1$ & $9.4264 e-1$ & $9.8332 e-1$ \\
\hline WFG2 & 15 & $7.6722 e-1$ & $9.3104 e-1$ & $6.7549 e-1$ & $9.1018 e-1$ & $5.4736 e-1$ & $8.0398 e-1$ & $9.7373 e-1$ & $9.7782 e-1$ \\
\hline WFG3 & 5 & $2.5974 e-2$ & $6.7116 e-1$ & $5.9385 e-1$ & $7.1066 e-1$ & $6.7204 e-1$ & $6.6812 e-1$ & $7.2408 e-1$ & $6.9653 e-1$ \\
\hline WFG3 & 10 & $3.7874 e-1$ & $5.6384 e-1$ & $2.2239 e-1$ & $6.0532 e-1$ & $2.7517 e-1$ & $2.5414 e-1$ & $7.3015 e-1$ & $6.2796 e-1$ \\
\hline WFG3 & 15 & $4.6421 e-1$ & $6.9188 e-1$ & $3.6466 e-1$ & $7.3573 e-1$ & $3.2567 e-1$ & $6.5152 e-1$ & $7.5401 e-1$ & $7.0778 e-1$ \\
\hline WFG4 & 5 & $7.4868 e-1$ & $7.5476 e-1$ & $6.4367 e-1$ & $7.4082 e-1$ & 6.603 & 7 . & -1 & $.5858 e-1$ \\
\hline WFG4 & 10 & $8.3259 e-1$ & $8.2460 e-1$ & $3.7213 e-1$ & $8.6530 e-1$ & $6.2085 e-1$ & $7.8810 e-1$ & $6.7389 e-1$ & $8.6804 e-1$ \\
\hline WFG4 & 15 & $7.6663 e-1$ & $8.7028 e-1$ & $1.6598 e-1$ & $7.8689 e-1$ & $3.3596 e-1$ & $8.6369 e-1$ & $6.1921 e-1$ & $9.0693 e-1$ \\
\hline WFG5 & 5 & $7.3031 e-1$ & $7.1458 e-1$ & $6.4534 e-1$ & $7.2793 e-1$ & $6.1046 e$ & $7.0839 e$ & $6.8516 e-1$ & $7.2568 e-1$ \\
\hline WFG5 & 10 & $8.4286 e-1$ & $7.9010 e-1$ & $3.7997 e-1$ & $8.4800 e-1$ & $5.5075 e-1$ & $6.8552 e-1$ & $6.1423 e-1$ & $8.3440 e-1$ \\
\hline WFG5 & 15 & $8.4490 e-1$ & $7.7143 e-1$ & $1.1919 e-1$ & $7.8855 e-1$ & $2.1399 e-1$ & $3.9362 e-1$ & $5.1556 e-1$ & $8.5625 e-1$ \\
\hline WFG6 & 5 & $7.3194 e-1$ & $7.3388 e-1$ & $5.4052 e-1$ & $7.2387 e-1$ & $6.5647 e-1$ & $7.1624 e-1$ & $6.8634 e-1$ & $7.3791 e-1$ \\
\hline WFG6 & 10 & $8.6056 e-1$ & $8.2632 e-1$ & $1.9400 e-1$ & $8.5875 e-1$ & $6.2132 e-1$ & $7.5777 e-1$ & $6.0675 e-1$ & $8.5307 e-1$ \\
\hline WFG6 & 15 & $8.6801 e-1$ & $8.4484 e-1$ & $9.4497 e-2$ & $8.5336 e-1$ & 32801 & $4.4625 e-1$ & $5.1359 e-1$ & $8.9315 e-1$ \\
\hline WFG7 & 5 & $7.8637 e-1$ & $7.8067 e-1$ & $6.3867 e-1$ & $7.7461 e-1$ & $7.6659 e-1$ & $7.6365 e-1$ & $7.5484 e-1$ & $7.9304 e-1$ \\
\hline WFG7 & 10 & $8.8809 e-1$ & $8.9075 e-1$ & $2.3503 e-1$ & $9.1733 e-1$ & $7.4944 e-1$ & $8.5089 e-1$ & $6.6219 e-1$ & $9.2941 e-1$ \\
\hline WFG7 & 15 & $7.2147 e-1$ & $9.2918 e-1$ & $1.2338 e-1$ & $8.3698 e-1$ & $4.2995 e-1$ & $9.1768 e-1$ & $6.0513 e-1$ & $9.7162 e-1$ \\
\hline WFG8 & 5 & $6.4019 e-1$ & $6.7275 e-1$ & $5.0839 e-1$ & $6.5130 e-1$ & $3.5919 e-1$ & $6.3295 e-1$ & $6.1111 e-1$ & $6.6159 e-1$ \\
\hline WFG8 & 10 & $6.0051 e-1$ & $8.0185 e-1$ & $7.3417 e-2$ & $7.8469 e-1$ & $4.6721 e-1$ & $7.0977 e-1$ & $4.4208 e-1$ & $7.4075 e-1$ \\
\hline WFG8 & 15 & $3.8537 e-1$ & $8.7264 e-1$ & $3.4644 e-1$ & $7.3358 e-1$ & $2.9260 e-1$ & $8.6234 e-1$ & $3.5437 e-1$ & $8.9873 e-1$ \\
\hline WFG9 & 5 & $6.8336 e-1$ & $6.7929 e-1$ & 5.887 & $6.4825 e-1$ & $3.8392 e-1$ & $6.3831 e-1$ & $6.3829 e-1$ & $6.6130 e-1$ \\
\hline WFG9 & 10 & $7.0854 e-1$ & $7.4727 e-1$ & $1.5646 e-1$ & $7.3520 e-1$ & $5.1312 e-1$ & $5.2354 e-1$ & $5.6358 e-1$ & $7.3830 e-1$ \\
\hline WFG9 & 15 & $6.1502 e-1$ & $7.1075 e-1$ & $6.0542 e-2$ & $7.1895 e-1$ & $2.0338 e-1$ & $2.4844 e-1$ & $4.4957 e-1$ & $7.2111 e-1$ \\
\hline
\end{tabular}

can be attributed to the fact that the PFs of the WFG test suite are irregular, discontinued or mixed, and scaled with different ranges in each objective. Therefore, well-distributed weight vectors/reference points cannot guarantee a good distribution of the obtained solutions. Note, however, that RVEA, which also uses the reference yectors to guide the search, performs better than MOEA/D, MOEA/DD, NSGA-III, and MOMBI-II. This is perhaps because the use of angle information helps RVEA to alleviate this issue to a certain degree.

The HV values are given in Table 7. From Table 7, AnD and SPEA2+SDE achieve the best and second best overall performance, respectively. Specifically, $\mathrm{AnD}$ produces 14 best results and three second best results out of 27 test problems, and SPEA2+SDE has three best results and eight second best results. It is also observed that AnD reaches the best performance on WFG2, WFG4, WFG6, and WFG7. For MOMBI-II, Two_Arch2, RVEA, and SPEA2+SDE, they exhibit the best overall performance on WFG1, WFG3, WFG5, and WFG8, respectively. 


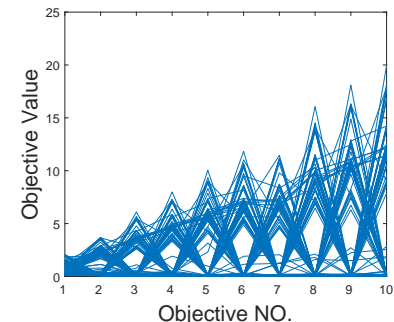

(a) RVEA

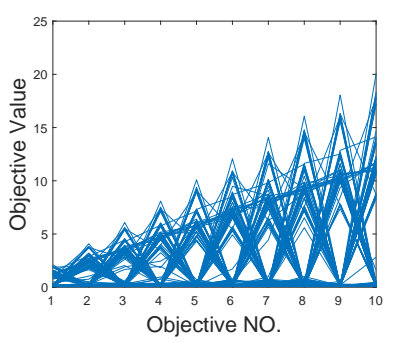

(d) NSGA-III

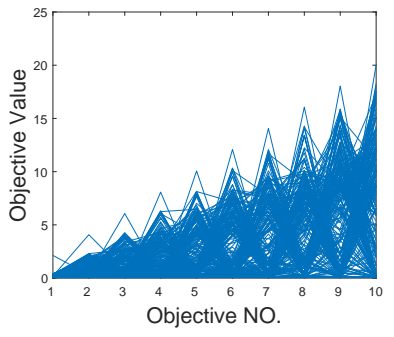

(b) SPEA2+SDE

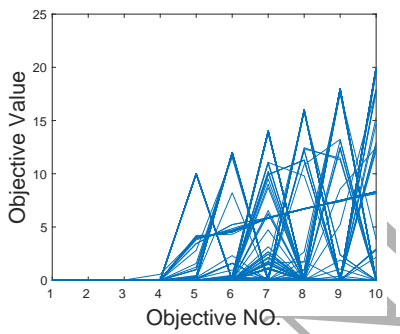

(e) MOMBI-II

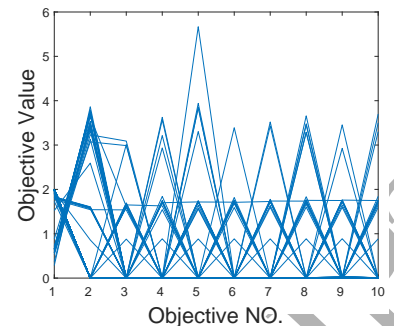

(c) MOEA/D

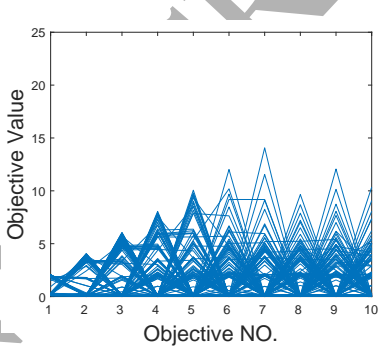

(f) MOEA/DD

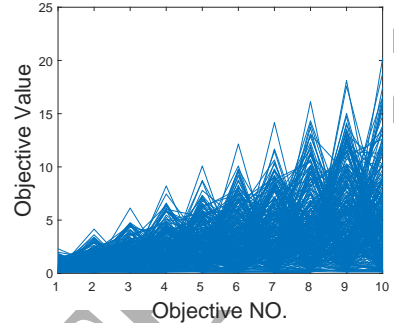

(g) Two_Arch2

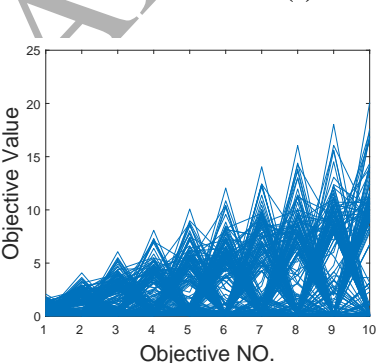

(h) $\mathrm{AnD}$

Figure 6: The final solution sets of the eight compared algorithms on WFG4 with ten objectives by parallel coordinates.

With regard to WFG9, AnD and SPEA2+SDE are the two best algorithms.

With the aim of revealing more details of the eight compared algorithms, their results on both WFG4 and WFG7 with ten objectives are presented by parallel coordinates in Figs. 6 and 7, respectively. From Fig. 6, one can see that MOEA/D and MOEA/DD have relatively poor distributions. It might be because they lack a normalization procedure before the evaluation of an individual. As for RVEA and MOMBI-II, the former fails to cover the seventh objective well, while the latter is 


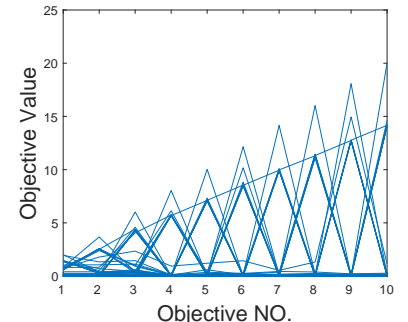

(a) RVEA

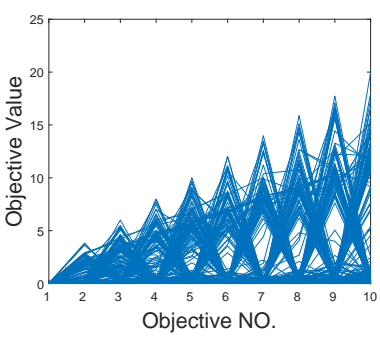

(d) NSGA-III

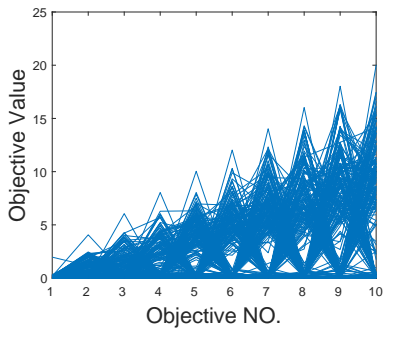

(b) SPEA2+SDE

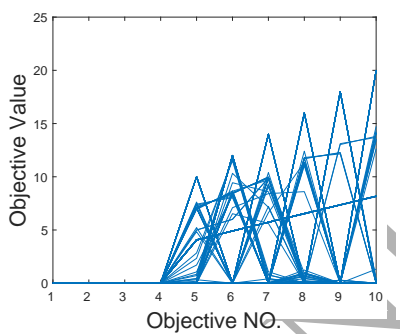

(e) MOMBI-II

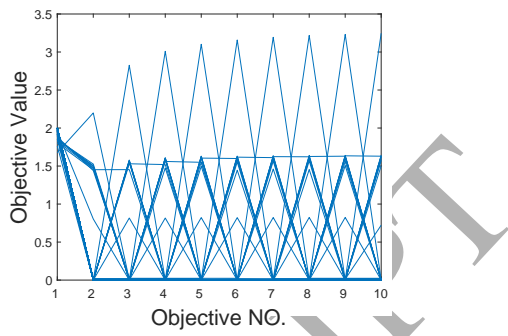

(c) MOEA/D

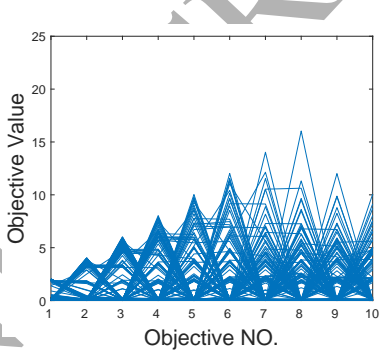

(f) MOEA/DD

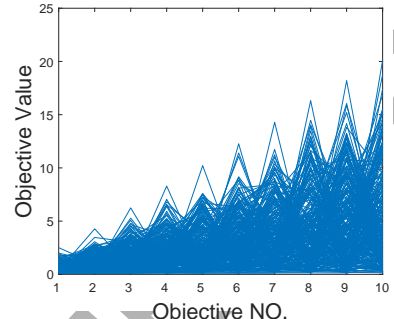

(g) Two_Arch2

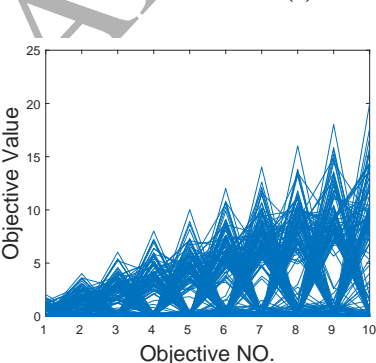

(h) $\mathrm{AnD}$

Figure 7: The final solution sets of the eight compared algorithms on WFG7 with ten objectives by parallel coordinates.

unable to cover the first four objectives well. In terms of SPEA2+SDE, NSGA-III, Two_Arch2, and AnD, all of them can cover the whole PF. The difference between them is that the results derived from SPEA2+SDE, NSGA-III, and Two_Arch2 concentrate mainly on the boundary or the middle parts of the PF, while in AnD, the results can spread out the whole PF very well. A similar phenomena can also be observed in Fig. 7. AnD still has the best distribution. Note that NSGA-III fails to cover the first objective well. 


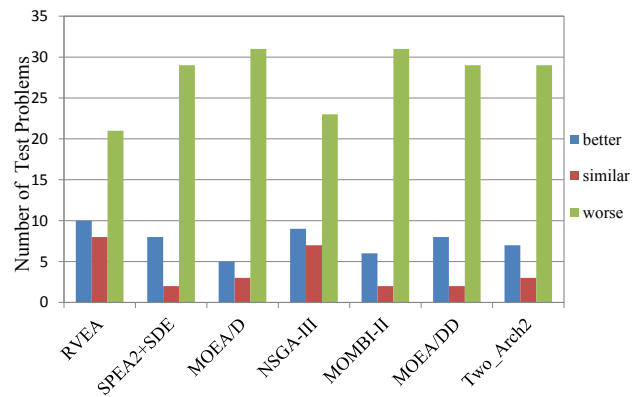

(a) IGD

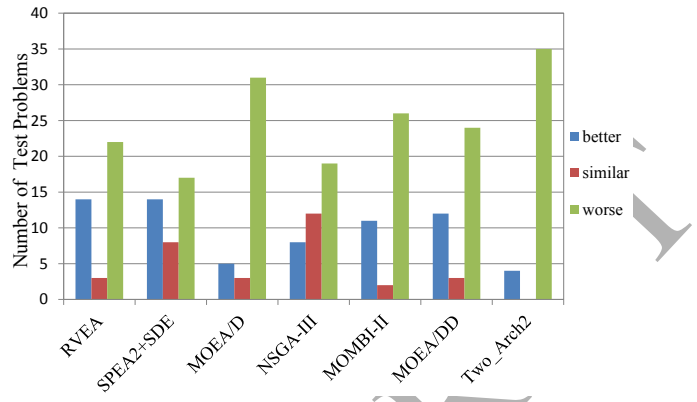

(b) $\mathrm{HV}$

Figure 8: Wilcoxon rank-sum test between $\mathrm{AnD}$ and its seven competitors (i.e., RVEA, SPEA2+SDE, MOEA/D, NSGA-III, MOMBI-II, MOEA/DD, and Two_Arch2) on all the test problems (including the DTLZ and WFG test suites) in terms of IGD and HV "better", "similar" and "worse" mean that a competitor performs better than, similar to, and worse than AnD, respectively.

\subsubsection{Discussion}

To analyze the overall performance on both the DTLZ and WFG test suites, the Wilcoxon rank-sum test was implemented between AnD and the other seven MaOEAs in terms of both the IGD and HV metrics. The statistical test results are presented in Fig. 8. Fig. 8(a) gives the comparison results in terms of IGD. From Fig. 8(a), we can see that AnD outperforms RVEA, SPEA2+SDE, MOEA/D, NSGA-III, MOMBI-II, MOEA/DD, and Two_Arch2 on 21, 29, 31, 23, 31, 29, and 29 test problems, respectively, while it loses on 10, eight, five, nine, six, eight, and seven test problems. The comparison results for HV are shown in Fig. 8(b). As shown in Fig. 8(b), AnD performs better than RAVE, SPEA2+SDE, MOEA/D, NSGA-III, MOMBI-II,MOEA/DD, and Two_Arch2 on 22, 17, 31, 19, 26, 24, and 35 test problems, respectively, but performs worse on 14, 14, five, eight, 11, 12, and four test problems. Thus, we can conclude that AnD is able to obtain better overall performance compared with the seven competitors in terms of both IGD and $\mathrm{HV}$.

Furthermore, the Friedman test was also implemented on all the test problems in terms of both IGD and HV. In the Friedman test, the smaller the ranking, the better the performance of the algorithm. From Fig. 9, it is evident that AnD has the smallest ranking in terms of both IGD and HV, followed by RVEA and SPEA2+SDE. RVEA ranks the third and second best in terms of IGD and HV, respectively. SPEA2+SDE works the second and third best in terms of IGD and 


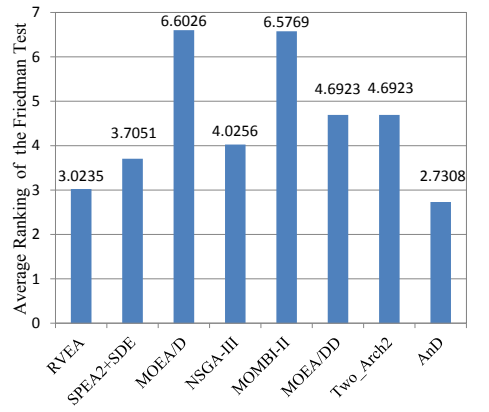

(a) IGD

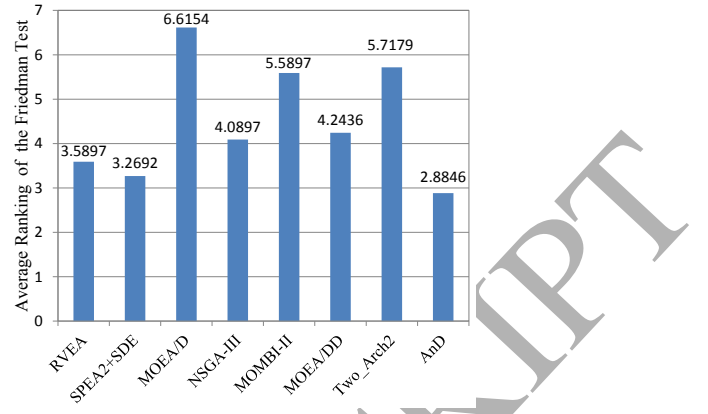

(b) $\mathrm{HV}$

Figure 9: Friedman test between $\mathrm{AnD}$ and its seven competitors (i.e., RVEA, SPEA2+SDE, MOEA/D, NSGA-III, MOMBI-II, MOEA/DD, and Two_Arch2) on all the test problems (including the DTLZ and WFG test suites) in terms of IGD and HV. The smaller the ranking, the better the performance of an algorithm.

$\mathrm{HV}$, respectively. Theses results indicate that the algorithm with either shift-based density estimation (i.e., SPEA2+SDE) or angle information (i.e., RVEA) is more suitable for solving MaOPs. Moreover, the algorithm with these two elements (i.e., AnD) achieves the best performance, which verifies the main motivation of this paper.

\subsection{Constrained MaOPs}

One may be interested in whether AnD can be applied to solve constrained MaOPs, which are frequently encountered in real-world applications. To answer this question, AnD was extended to cope with this kind of optimization problem, and the resultant algorithm is called C-AnD.

The constraint-handling technique of C-AnD is inspired by the feasibility rule [7], which is a well-known constraint-handling technique for constrained single-objective optimization problems. Firstly, we compute the degree of constraint violation for each individual:

$$
C V(\mathbf{x})=\sum_{j=1}^{J} \max \left\{0, g_{j}(\mathbf{x})\right\}+\sum_{k=1}^{K}\left|h_{k}(\mathbf{x})\right| .
$$

where $g_{j} \geq 0$ and $h_{j}=0$ denote the $j$ th inequality constraint and the $j$ th equality constraint, respectively, and $J$ and $K$ are the number of inequality constraints and equality constraints, respectively. Subsequently, the number of feasible solutions 
Table 8: Mean and standard deviation of the IGD and HV values on C1-DTLZ1, C2-DTLZ2, and C3-DTLZ4. The better result between C-AnD and C-NSGA-III on each test problem is highlighted in Gray.

\begin{tabular}{|c|c|c|c|}
\hline IGD & $m$ & C-NSGA-III & C-AnD \\
\hline C1-DTLZ1 & 5 & $5.8093 e-2(5.60 e-3)$ & $5.4026 e-2(5.46 e-3)$ \\
\hline C1-DTLZ1 & 10 & $1.2436 e-1(4.98 e-3)$ & $1.1237 e-1(3.26 e-3)$ \\
\hline C1-DTLZ1 & 15 & $2.0774 e-1(1.13 e-2)$ & $1.7216 e-1(2.01 e-3)$ \\
\hline \multicolumn{4}{|c|}{ - - - - - - - - - - - - - - - - - - - - - - - - - - - - - - - - } \\
\hline C2-DTLZ2 & 5 & $1.8916 e-1(7.44 e-2)$ & $1.5010 e-1(6.73 e-3)$ \\
\hline C2-DTLZ2 & 10 & $3.7888 e-1(1.20 e-1)$ & $2.6826 e-1(5.60 e-2)$ \\
\hline C2-DTLZ2 & 15 & $7.2215 e-1(1.56 e-1)$ & $2.6633 e-1(1.62 e-1)$ \\
\hline C3-DTLZ4 & 5 & $2.8139 e-1(2.04 e-2)$ & $2.8605 e-1(1.40 e-2)$ \\
\hline C3-DTLZ4 & 10 & $5.7678 e-1(6.60 e-2)$ & $5.4039 e-1(1.34 e-2)$ \\
\hline C3-DTLZ4 & 15 & $1.1089 e+0(2.72 e-1)$ & $7.3981 e-1(2.88 e-3)$ \\
\hline HV & $m$ & \multicolumn{2}{|l|}{ C-NSGA-III } \\
\hline C1-DTLZ1 & 5 & $9.7255 e-1(2.73 e-3)$ & \multirow{3}{*}{$\begin{array}{l}9.7770 e-1(1.90 e-3) \\
9.8803 e-1(1.66 e-2) \\
9.9054 e-1(1.19 e-2)\end{array}$} \\
\hline C1-DTLZ1 & 10 & $9.8800 e-1(1.05 e-2)$ & \\
\hline C1-DTLZ1 & 15 & $9.5938 e-1(3.76 e-2)$ & \\
\hline \multicolumn{4}{|c|}{ 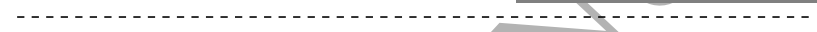 } \\
\hline C2-DTLZ2 & 5 & $7.3902 e-1(4.53 e-2)$ & \multirow{3}{*}{$\begin{array}{l}7.4493 e-1(3.45 e-3) \\
8.7759 e-1(1.46 e-2) \\
9.0674 e-1(1.65 e-1)\end{array}$} \\
\hline C2-DTLZ2 & 10 & $8.3045 e-1(7.04 e-2)$ & \\
\hline C2-DTLZ2 & 15 & $5.9223 e-1(2.35 e-1)$ & \\
\hline \multicolumn{4}{|c|}{ 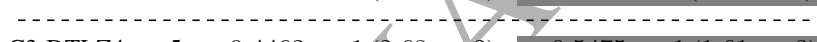 } \\
\hline C3-DTLZ4 & 5 & $9.4462 e-1(2.68 e-2)$ & \multirow{3}{*}{$\begin{array}{l}9.5475 e-1(1.61 e-3) \\
9.9909 e-1(1.08 e-4) \\
9.9991 e-1(1.84 e-5)\end{array}$} \\
\hline C3-DTLZ4 & 10 & $9.9877 e-1(2.51 e-3)$ & \\
\hline C3-DTLZ4 & 15 & $9.5658 e-1(3.70 e-2)$ & \\
\hline
\end{tabular}

in the union population $\mathcal{U}_{t}$ is calculated. If the number of feasible solutions is larger than $N$, then Algorithm 2 is triggered to select $N$ feasible solutions for the next generation from all the feasible solutions. Otherwise, we sort the individuals in $\mathcal{U}_{t}$ according to their degree of constraint violations, and then choose $N$ individuals with the smallest degree of constraint violations for the next generation.

Overall, the implementation of C-AnD is simple. The performance of C-AnD was compared with that of C-NSGA-III [21], which is the constrained version of NSGA-III, on three representative constrained MaOPs, namely C1-DTLZ1, C2-DTLZ2, and C3-DTLZ4 with five, 10, and 15 objectives. Both C-AnD and C-NSGA-III were run 20 times independently for each test problem. In each run, the maximum number of FEs was set to 180,000 for C1-DTLZ1, and 90,000 for C2-DTLZ2 and C3-DTLZ4. The results are summarized in Table 8.

From Table 8 , it can be seen that C-AnD beats C-NSGA-III on all the test problems except C3-DTLZ4 with five objectives in terms of IGD. Therefore, C$\mathrm{AnD}$ is also a simple and effective algorithm for constrained many-objective optimization. It is worth noting that there are no reference points in C-AnD; thus 
C-AnD does not experience the degeneration of reference points as in constrained decomposition-based approaches.

\section{Conclusion}

In this paper, an alternative algorithm for dealing with MaOPs, named AnD, has been proposed. AnD not only has a simple structure, but also is free from the use of the Pareto-dominance relation, weight vectors or reference points, and indicators. The main characteristic of $\mathrm{AnD}$ is that it makes use of two strategies (i.e., angle-based selection and shift-based density estimation) to delete poor individuals one by one during the environmental selection,

The aim of the angle-based selection strategy is to maintain the diversity of search directions. It identifies a pair of individuals with the minimum vector angle, which means that these two individuals search in the most similar directions. Subsequently, shift-based density estimation is conducted to differentiate them by considering both diversity and convergence, and to remove the inferior one. We validated that these two strategies play very important roles and are indispensable in AnD. In addition, we compared AnD with seven state-of-the-art MaOEAs for solving MaOPs with up to 15 objectives in the DTLZ and WFG test suites. The results indicate that, overall, $\mathrm{AnD}$ achieves the best performance in terms of both IGD and HV. AnD was further extended to solve constrained MaOPs and the results verify its effectiveness

In the future, we will apply AnD to solve some unconstrained and constrained MaOPs in the fields of engineering, such as automotive lightweight design and adaptive walking of humanoid robots. Another promising research direction is to combine AnD with other kinds of MaOEAs, such as Pareto-based, decompositionbased, and indicator-based approaches.

The Matlab source code of AnD can be downloaded from Y. Wang's homepage: http://www.escience.cn/people/yongwang 1/index.html

\section{References}

[1] S. F. Adra, P. J. Fleming, Diversity management in evolutionary manyobjective optimization, IEEE Transactions on Evolutionary Computation 15 (2) (2011) 183-195.

[2] J. Bader, E. Zitzler, HypE: An algorithm for fast hypervolume-based manyobjective optimization, Evolutionary Computation 19 (1) (2011) 45-76. 
[3] X. Cai, Z. Yang, Z. Fan, Q. Zhang, Decomposition-based-sorting and anglebased-selection for evolutionary multiobjective and many-objective optimization, IEEE Transactions on Cybernetics 47 (9) (2017) 2824-2837.

[4] R. Cheng, Y. Jin, M. Olhofer, B. Sendhoff, A reference vector guided evolutionary algorithm for many-objective optimization, IEEE Transactions on Evolutionary Computation 20 (5) (2016) 773-791.

[5] C. A. Coello Coello, G. B. Lamont, D. A. Van Veldhuizen, et al., Evolutionary Algorithms for Solving Multi-objective Problems, vol. 5, Springer, 2007.

[6] D. W. Corne, J. D. Knowles, Techniques for highly multiobjective optimisation: Some nondominated points are better than others, in: Proceedings of the 9th Annual Conference on Genetic and Evolutionary Computation, 773-780, 2007.

[7] K. Deb, An efficient constraint handling method for genetic algorithms, Computer Methods in Applied Mechanics and Engineering 186 (2) (2000) 311-338.

[8] K. Deb, H. Jain, An evolutionary many-objective optimization algorithm using reference-point-based nondominated sorting approach, Part I: Solving problems with box constraints., IEEE Transactions on Evolutionary Computation 18 (4) (2014) 577-601.

[9] K. Deb, A. Pratap, S. Agarwal, T. Meyarivan, A fast and elitist multiobjective genetic algorithm: NSGA-II, IEEE Transactions on Evolutionary Computation 6 (2) (2002) 182-197.

[10] K. Deb, L. Thiele, M. Laumanns, E. Zitzler, Scalable test problems for evolutionary multiobjective optimization, Evolutionary Multiobjective Optimization. Theoretical Advances and Applications (2005) 105-145.

[11] R. Denysiuk, A. Gaspar-Cunha, Multiobjective evolutionary algorithm based on vector angle neighborhood, Swarm and Evolutionary Computation 37 (2017) 45-57.

[12] M. Emmerich, N. Beume, B. Naujoks, An EMO algorithm using the hypervolume measure as selection criterion., in: EMO, vol. 3410, Springer, 62-76, 2005. 
[13] G. Fillipe, C. Felipe, Preference-guided evolutionary algorithms for manyobjective optimization, Information Sciences 329 (2016) 236-255.

[14] A. R. d. Freitas, P. J. Fleming, F. G. Guimaräesc, Aggregation trees for visualization and dimension reduction in many-objective optimization, Information Sciences 298 (2015) 288-314.

[15] Z. He, G. G. Yen, Many-objective evolutionary algorithms based on coordinated selection strategy, IEEE Transactions on Evolutionary Computation 21 (2) (2017) 220-233.

[16] R. Hernández Gómez, C. A. Coello Coello, Improved metaheuristic based on the R2 indicator for many-objective optimization, in: Proceedings of the 2015 Annual Conference on Genetic and Evolutionary Computation, ACM, 679-686, 2015.

[17] S. Huband, P. Hingston, L. Barone, L. While, A review of multiobjective test problems and a scalable test problem toolkit, IEEE Transactions on Evolutionary Computation 10 (5) (2006) 477-506.

[18] E. J. Hughes, MSOPS-II: A general-purpose many-objective optimiser, in: IEEE Congress on Evolutionary Computation (CEC 2007), IEEE, 3944 3951, 2007.

[19] H. Ishibuchi, N. Akedo, Y. Nojima, Behavior of multiobjective evolutionary algorithms on many-objective knapsack problems, IEEE Transactions on Evolutionary Computation 19 (2) (2015) 264-283.

[20] H. Ishibuchi, H. Masuda, Y. Tanigaki, Y. Nojima, Review of coevolutionary developments of evolutionary multi-objective and many-objective algorithms and test problems, in: 2014 IEEE Symposium on Computational Intelligence in Multi-Criteria Decision-Making, IEEE, 178-184, 2014.

[21] H. Jain, K. Deb, An evolutionary many-objective optimization algorithm using reference-point based nondominated sorting approach, Part II: Handling constraints and extending to an adaptive approach, IEEE Transactions on Evolutionary Computation 18 (4) (2014) 602-622.

[22] S. Jiang, S. Yang, A strength Pareto evolutionary algorithm based on reference direction for multiobjective and many-Objective optimization, IEEE Transactions on Evolutionary Computation 21 (3) (2017) 329-346. 
[23] M. Laumanns, L. Thiele, K. Deb, E. Zitzler, Combining convergence and diversity in evolutionary multiobjective optimization, Evolutionary Computation 10 (3) (2002) 263-282.

[24] B. Li, J. Li, K. Tang, X. Yao, Many-objective evolutionary algorithms: A survey, ACM Computing Surveys (CSUR) 48 (1) (2015) 13.

[25] B. Li, K. Tang, J. Li, X. Yao, Stochastic ranking algorithm for manyobjective optimization based on multiple indicators, IEEE Transactions on Evolutionary Computation 20 (6) (2016) 924-938.

[26] K. Li, K. Deb, Q. Zhang, S. Kwong, An evolutionary many-objective optimization algorithm based on dominance and decomposition, IEEE Transactions on Evolutionary Computation 19 (5) (2015) 694-716.

[27] M. Li, S. Yang, X. Liu, Shift-based density estimation for Pareto-based algorithms in many-objective optimization, IEEE Transactions on Evolutionary Computation 18 (3) (2014) 348-365.

[28] H. L. Liu, L. Chen, Q. Zhang, K. Deb, Adaptively allocating search effort in challenging many-objective optimization problems, IEEE Transactions on Evolutionary Computation, 2018, in press, DOI: 10.1109/TEVC.2017.2725902 .

[29] P. Monalisa, S. Sriparna, B. Sanghamitra, DECOR: Differential evolution using clustering based objective reduction for many-objective optimization, Information Sciences 423 (2018) 200-218.

[30] H. K. Singh, A. Isaacs, T. Ray, A Pareto corner search evolutionary algorithm and dimensionality reduction in many-objective optimization problems, IEEE Transactions on Evolutionary Computation 15 (4) (2011) 539_ 556.

[31] Y. Tian, R. Cheng, X. Zhang, Y. Jin, PlatEMO: A MATLAB platform for evolutionary multi-Objective optimization, IEEE Computational Intelligence Magzine 12 (4) (2017) 73-87.

[32] H. Trautmann, T. Wagner, D. Brockhoff, R2-EMOA: Focused multiobjective search using R2-indicator-based selection, in: International Conference on Learning and Intelligent Optimization, Springer, 70-74, 2013. 
[33] M. Wagner, F. Neumann, A fast approximation-guided evolutionary multiobjective algorithm, in: Conference on Genetic and Evolutionary Computation, 687-694, 2013.

[34] G. Wang, H. Jiang, Fuzzy-dominance and its application in evolutionary many objective optimization, in: International Conference on Computational Intelligence and Security Workshops (CISW 2007), IEEE, 195-198, 2007.

[35] H. Wang, L. Jiao, X. Yao, Two_Arch2: An improved two-archive algorithm for many-objective optimization, IEEE Transactions on Evolutionary Computation 19 (4) (2015) 524-541.

[36] J. Wang, W. Zhang, J. Zhang, Cooperative differential evolution with multiple populations for multiobjective optimization, IEEE Transactions on Cybernetics 46 (12) (2016) 2848-2861.

[37] R. Wang, R. C. Purshouse, P. J. Fleming, Preference-inspired coevolutionary algorithms for many-objective optimization, IEEE Transactions on Evolutionary Computation 17 (4) (2013) 474-494.

[38] R. Wang, Q. Zhang, T. Zhang, Decomposition-based algorithms using Pareto adaptive scalarizing methods, IEEE Transactions on Evolutionary Computation 20 (6) (2016) 821-837.

[39] R. Wang, Z. Zhou, H. Ishibuchi, T. Liao, T. Zhang, Localized weighted sum method for many-objective optimization, IEEE Transactions on Evolutionary Computation 22 (1) (2018) 3-18.

[40] Y. Xiang, Y. Zhou, M. Li, Z. Chen, A vector angle-based evolutionary algorithm for unconstrained many-Objective Optimization, IEEE Transactions on Evolutionary Computation 21 (1) (2017) 131-152.

[41] Y. Yuan, H. Xu, B. Wang, B. Zhang, X. Yao, Balancing convergence and diversity in decomposition-based many-objective optimizers, IEEE Transactions on Evolutionary Computation 20 (2) (2016) 180-198.

[42] Q. Zhang, H. Li, MOEA/D: A multiobjective evolutionary algorithm based on decomposition, IEEE Transactions on Evolutionary Computation 11 (6) (2007) 712-731. 
[43] X. Zhang, Y. Tian, Y. Jin, A knee point-driven evolutionary algorithm for many-objective optimization, IEEE Transactions on Evolutionary Computation 19 (6) (2015) 761-776.

[44] A. Zhou, B.-Y. Qu, H. Li, S.-Z. Zhao, P. N. Suganthan, Q. Zhang, Multiobjective evolutionary algorithms: A survey of the state of the art, Swarm and Evolutionary Computation 1 (1) (2011) 32-49.

[45] E. Zitzler, S. Künzli, Indicator-based selection in multiobjective search, in: International Conference on Parallel Problem Solying from Nature, Springer, 832-842, 2004.

[46] E. Zitzler, M. Laumanns, L. Thiele, SPEA2: Improving the strength Pareto evolutionary algorithm for multiobjective optimization, Proceedings of Evolutionary Methods for Design, Optimization and Control with Applications to Industrial Problems, EUROGEN'2001 (2001) 95-100.

[47] E. Zitzler, L. Thiele, Multiobjective optimization using evolutionary algorithms - A comparative case study, in: International Conference on Parallel Problem Solving from Nature, Springer, 292-301, 1998.

[48] X. Zou, Y. Chen, M. Liu, L. Kang, A new evolutionary algorithm for solving many-objective optimization problems, IEEE Transactions on Systems, Man, and Cybernetics, Part B (Cybernetics) 38 (5) (2008) 1402-1412. 